\title{
ASYMPTOTICS FOR SOME POLYNOMIAL PATTERNS IN THE PRIMES
}

\author{
PIERRE-YVES BIENVENU
}

Abstract. We prove asymptotic formulae for sums of the form

$$
\sum_{n \in \mathbb{Z}^{d} \cap K} \prod_{i=1}^{t} F_{i}\left(\psi_{i}(n)\right),
$$

where $K$ is a convex body, each $F_{i}$ is either the von Mangoldt function or the representation function of a quadratic form, and $\Psi=\left(\psi_{1}, \ldots, \psi_{t}\right)$ is a system of linear forms of finite complexity. When all the functions $F_{i}$ are equal to the von Mangoldt function, we recover a result of Green and Tao, while when they are all representation functions of quadratic forms, we recover a result of Matthiesen. Our formulae imply asymptotics for some polynomial patterns in the primes. Specifically, they describe the asymptotic behaviour of the number of $k$-term arithmetic progressions of primes whose common difference is a sum of two squares.

The article combines ingredients from the work of Green and Tao on linear equations in primes and that of Matthiesen on linear correlations amongst integers represented by a quadratic form. To make the von Mangoldt function compatible with the representation function of a quadratic form, we provide a new pseudorandom majorant for both - an average of the known majorants for each of the functions - and prove that it has the required pseudorandomness properties.

\section{INTRODUCTION}

In a celebrated article [7], Green and Tao proved the following.

Theorem 1.1. The set $\mathcal{P}$ of primes contains arbitrarily long arithmetic progressions.

This result has been strengthened and generalised in a number of ways over the last decade. One crucial such strengthening was achieved by Green and Tao [8], when they proved the existence of prime solutions to a large class of systems of linear equations. Moreover, they provided asymptotics for the number of such solutions. Among other things, they proved an asymptotic for the number of $k$-term arithmetic progressions in the primes up to $N$. Their result was conditional on two conjectures that Green, Tao and Ziegler later proved completely [9, 10].

Applying the same general method as Green and Tao, Matthiesen obtained similar results with the divisor function [15] or the representation function of quadratic forms [16] instead of the von Mangoldt function.

Date: October 15, 2018. 
In this paper, we state and prove a theorem (Theorem 1.2) which encompasses both Green and Tao's and Matthiesen's results. It implies asymptotics for some - admittedly very specific - polynomial patterns. For instance, Corollary 1.3 gives the asymptotic for the number of $k$-term arithmetic progressions of primes whose common difference is a sum of two squares, where each such progression is counted as many times as the common difference is represented a sum of two squares.

Since the first submission of our paper, Tao and Ziegler [19] obtained asymptotics for a much larger class of polynomial progressions, improving upon their earlier work [17] that gave only lower bounds. The methods they develop are vastly more intricate than ours, but there exist a number of interesting equations involving primes and sums of two squares which belong to the scope of our theorem but not to theirs.

Acknowledgements. The author thanks his supervisor Julia Wolf for useful conversations, guidance and reading many drafts, Sean Prendiville for suggesting this problem, and Sam Chow and Andy Corbett for useful remarks.

1.1. Preliminaries. We require a few definitions and some notation to be able to state our theorem. The von Mangoldt function $\Lambda$ is defined on $\mathbb{N}$ by setting $\Lambda(n)=\log p$ if $n$ is a power of a prime $p$ and $\Lambda(n)=0$ otherwise. We define for any integer $q$ the local von Mangoldt function on $\mathbb{Z}$ by

$$
\Lambda_{q}(n)=\frac{q}{\phi(q)} 1_{(n, q)=1}
$$

where $\phi$ is the Euler totient function defined by $\phi(q)=\left|(\mathbb{Z} / q \mathbb{Z})^{*}\right|$ and $(n, q)$ is the greatest common divisor (gcd) of $n$ and $q$. In fact, $\Lambda_{q}$ can naturally be defined on $\mathbb{Z} / k q \mathbb{Z}$ for any $k \in \mathbb{N}$. Observe the use of the symbol $1_{P}$ for a proposition $P$, which means 1 if $P$ is true and 0 otherwise.

Let $d, t \geq 1$ be integers. An affine-linear form $\psi$ on $\mathbb{Z}^{d}$ is a polynomial in $d$ variables of degree at most 1 with integer coefficients. We denote by $\dot{\psi}$ its linear part; then $\psi=\dot{\psi}+\psi(0)$.

If $\psi_{1}, \ldots, \psi_{t}$ are affine-linear forms, we say that $\Psi=\left(\psi_{1}, \ldots, \psi_{t}\right): \mathbb{Z}^{d} \rightarrow \mathbb{Z}^{t}$ is a system of affine-linear forms. It has finite complexity if no two of the forms are affinely dependent, i.e. for any $i \neq j$, the linear parts $\dot{\psi}_{i}$ and $\dot{\psi}_{j}$ are not proportional.

A binary quadratic form is a polynomial

$$
f(x, y)=a x^{2}+b x y+c y^{2}
$$

where $a, b$ and $c$ are integers. Its discriminant is $D=b^{2}-4 a c$. A positive definite binary quadratic form (abbreviated as PDBQF) is a binary quadratic form of negative discriminant. The representation function of $f$ is the arithmetic function defined by

$$
R_{f}(n)=\left|\left\{(x, y) \in \mathbb{Z}^{2} \mid f(x, y)=n\right\}\right| .
$$

For any integers $q$ and $\beta$, we let

$$
\rho_{f, \beta}(q)=\left|\left\{(x, y) \in[q]^{2} \mid f(x, y) \equiv \beta \bmod q\right\}\right| .
$$

We shall use the notation

$$
\mathbb{E}_{a \in A}=\frac{1}{|A|} \sum_{a \in A}
$$


to denote the averaging operator. We may also write $\mathbb{P}_{a \in X}(a \in A)$ for $|A| /|X|$, for finite sets $A \subset X$. The letter $p$ is reserved for primes, the set of which is denoted by $\mathcal{P}$; for instance $\prod_{p}$ implicitly means $\prod_{p \in \mathcal{P}}$.

The asymptotic parameter going to infinity is denoted by $N$. We use the symbols $X \sim Y$ to say that $X / Y$ tends to 1 as $N$ tends to infinity. We shall use $X=O(Y)$ to say that $X / Y$ is bounded and $X=o(Y)$ to say that $X / Y$ tends to 0 . Both $O$ and $o$ can be complemented with a subscript indicating the dependence of the implied constant or the implied decaying function. We also use $X \ll Y$, which is synonymous to $X=O(Y)$ and can be complemented by subscripts as well.

1.2. The main theorem. We are now ready to state our main theorem.

Theorem 1.2. Let $\Psi=\left(\psi_{1}, \ldots, \psi_{t+s}\right): \mathbb{Z}^{d} \rightarrow \mathbb{Z}^{t+s}$ be a system of affine-linear forms of finite complexity. Suppose that the coefficients of the linear part $\dot{\Psi}$ are bounded by some constant $L$. Let $K \subset[-N, N]^{d}$ be a convex body such that $\Psi(K) \subset[0, N]^{t+s}$. Let $f_{t+1}, \ldots, f_{t+s}$ be PDBQFs of discriminants $D_{j}<0$ for $j=t+1, \ldots, t+s$. Then

$$
\sum_{n \in \mathbb{Z}^{d} \cap K} \prod_{i=1}^{t} \Lambda\left(\psi_{i}(n)\right) \prod_{j=t+1}^{t+s} R_{f_{j}}\left(\psi_{j}(n)\right)=\beta_{\infty} \prod_{p} \beta_{p}+o\left(N^{d}\right)
$$

where

$$
\beta_{\infty}=\operatorname{Vol}(K) \prod_{j=t+1}^{t+s} \frac{2 \pi}{\sqrt{-D_{j}}}
$$

and

$$
\beta_{p}=\lim _{m \rightarrow+\infty} \mathbb{E}_{a \in\left(\mathbb{Z} / p^{m} \mathbb{Z}\right)^{d}} \prod_{i=1}^{t} \Lambda_{p}\left(\psi_{i}(a)\right) \prod_{j=t+1}^{t+s} \frac{\rho_{f_{j}, \psi_{j}(a)}\left(p^{m}\right)}{p^{m}}
$$

The error term is not effective (see [8, Sections 13 and 14] for a discussion) and the implied decaying function depends on $d, t, s, L$ and the discriminants.

Two important special cases arise when $s=0$ or $t=0$, that is, when the functions featuring are either all equal to the von Mangoldt function, or all representation functions. Then one of the products is trivial.

- When $s=0$, one immediately recovers the result of Green and Tao [8, Main Theorem]. Indeed, for $m \geq 1$, we have

$$
\mathbb{E}_{a \in\left(\mathbb{Z} / p^{m} \mathbb{Z}\right)^{d}} \prod_{i=1}^{t} \Lambda_{p}\left(\psi_{i}(a)\right)=\mathbb{E}_{a \in(\mathbb{Z} / p \mathbb{Z})^{d}} \prod_{i=1}^{t} \Lambda_{p}\left(\psi_{i}(a)\right)
$$

\footnotetext{
${ }^{1}$ Green and Tao 8 introduced the notion of size at scale $N$. One can check that the condition that the system has bounded size at scale $N$ is equivalent to the boundedness of the linear part together with the condition on the image of $K$.
} 
so that

$$
\beta_{p}=\mathbb{E}_{a \in(\mathbb{Z} / p \mathbb{Z})^{d}} \prod_{i=1}^{t} \Lambda_{p}\left(\psi_{i}(a)\right) .
$$

- When $t=0$, Theorem 1.2 boils down to the formula of Matthiesen [16, Theorem 1.1].

For each prime $p$, we call $\beta_{p}$ the local factor modulo $p$. The existence of the limit as $m$ tends to infinity that defines it is proven in Proposition A.1, the convergence of the infinite product $\prod_{p} \beta_{p}$ is a consequence of A.3.

Sometimes one can get an asymptotic even when the system has infinite complexity, but the asymptotic takes a completely different form then. For instance it is easy to see that

$$
\sum_{n \leq N} \Lambda(n) R(n) \sim 8 \sum_{\substack{p \leq N \\ p \equiv 1 \bmod 4}} \log p \sim 4 N
$$

by Fermat's theorem on sums of two squares and the prime number theorem in arithmetic progressions. We do not address such systems in this paper.

1.3. Progressions of step a sum of two squares in the primes. Here $R$ and $\rho$ (see Section 1.1) will implicitly refer to the form $f(x, y)=x^{2}+y^{2}$ whose discriminant is -4 . Our application concerns arithmetic progressions in the primes whose common difference is required to be a sum of two squares. It shows that the Green-Tao theorem (case $s=0$ of Theorem 1.2) holds not only for linear systems, but also for some - admittedly very specific - polynomial systems.

Corollary 1.3. Let $k \geq 1$ be an integer and

$$
L=\left\{(a, b, c) \in \mathbb{R}^{3} \mid 1 \leq a \leq a+(k-1)\left(b^{2}+c^{2}\right) \leq N\right\} .
$$

Let $\Psi=\left(\psi_{0}, \cdots, \psi_{k-1}\right) \in \mathbb{Z}[a, b, c]^{k}$ be the polynomial system defined by

$$
\psi_{i}(a, b, c)=a+i\left(b^{2}+c^{2}\right) .
$$

Then

$$
\sum_{n \in \mathbb{Z}^{3} \cap L} \prod_{i=0}^{k-1} \Lambda\left(\psi_{i}(n)\right)=\beta_{\infty} \prod_{p} \beta_{p}+o\left(N^{2}\right)
$$

with $\beta_{\infty}=\operatorname{Vol}(L)$ and

$$
\beta_{p}=\mathbb{E}_{n \in(\mathbb{Z} / p \mathbb{Z})^{3}} \prod_{i=0}^{k-1} \Lambda_{p}\left(\psi_{i}(n)\right) .
$$

As noted in the introduction, Corollary 1.3 now appears as a special case of a very recent result of Tao and Ziegler [19, Theorem 1.4]. However, our method can deal with the variant where $L$ is replaced by $[N] \times\left[\sqrt{N} \log ^{-A} N\right]^{2}$ for any constant $A>0$, thus the common difference of the progression is markedly smaller than the terms of the progression. Indeed, in the proof below, we reduce equation (11) to one involving a linear system, to which we apply [19, Theorem 1.3]. When one considers the system as a polynomial one, one cannot restrict $b$ and $c$ to such a small range. 
Proof of Corollary 1.3 assuming Theorem 1.2. We note that the left-hand side of equation (11) can be written as

$$
\sum_{(a, d) \in \mathbb{Z}^{2} \cap K} \Lambda(a) \Lambda(a+d) \cdots \Lambda(a+(k-1) d) R(d)
$$

where $K=\left\{(a, d) \in \mathbb{R}^{2} \mid 1 \leq a \leq a+(k-1) d \leq N\right\}$ is a convex body in $\mathbb{R}^{2}$. Applying Theorem 1.2 to this convex body and the system $(a, d) \mapsto(a, a+d, \ldots, a+(k-1) d, d)$, which is of finite complexity, we get

$$
\sum_{n \in \mathbb{Z}^{3} \cap L} \prod_{i=0}^{k-1} \Lambda\left(\psi_{i}(n)\right)=\beta_{\infty} \prod_{p} \beta_{p}+o\left(N^{2}\right)
$$

with $\beta_{\infty}=\pi \frac{N^{2}}{2(k-1)}$ and

$$
\beta_{p}=\lim _{m \rightarrow \infty} \mathbb{E}_{(a, d) \in\left(\mathbb{Z} / p^{m} \mathbb{Z}\right)^{2}} \frac{\rho_{d}\left(p^{m}\right)}{p^{m}}\left(\frac{p}{\phi(p)}\right)^{k} \prod_{i=0}^{k-1} 1_{(a+i d, p)=1}
$$

It is easy to see that $\operatorname{Vol}(L)=\beta_{\infty}$. It remains to prove that the local factors have the form (2). First,

$$
\mathbb{E}_{(a, d) \in\left(\mathbb{Z} / p^{m} \mathbb{Z}\right)^{2}} \frac{\rho_{d}\left(p^{m}\right)}{p^{m}} \prod_{i=0}^{k-1} 1_{(a+i d, p)=1}=\mathbb{E}_{(a, b, c) \in\left(\mathbb{Z} / p^{m} \mathbb{Z}\right)^{3}} \prod_{i=0}^{k-1} 1_{\left(a+i\left(b^{2}+c^{2}\right), p\right)=1}
$$

Now let $a \mapsto \tilde{a}$ be the canonical map $\mathbb{Z} / p^{m} \mathbb{Z} \rightarrow \mathbb{Z} / p \mathbb{Z}$. We notice that it is a $p^{m-1}$-to-1 map and that $\left(a+i\left(b^{2}+c^{2}\right), p\right)=1$ if and only if $\left(\tilde{a}+i\left(\tilde{b}^{2}+\tilde{c}^{2}\right), p\right)=1$. Hence

$$
\mathbb{E}_{(a, b, c) \in\left(\mathbb{Z} / p^{m} \mathbb{Z}\right)^{3}} \prod_{i=0}^{k-1} 1_{\left(a+i\left(b^{2}+c^{2}\right), p\right)=1}=\mathbb{E}_{(a, b, c) \in(\mathbb{Z} / p \mathbb{Z})^{3}} \prod_{i=0}^{k-1} 1_{\left(a+i\left(b^{2}+c^{2}\right), p\right)=1}
$$

does not depend on $m$ and the local factors are of the desired form.

Let us compute explicitly the local factors $\beta_{p}$. Suppose first that $p \geq k$. We remark that

$$
\beta_{p}=\left(\frac{p}{p-1}\right)^{k} \frac{1}{p} \sum_{a \in(\mathbb{Z} / p \mathbb{Z})^{*}}\left(1-\sum_{i=1}^{k-1} \mathbb{P}_{(b, c) \in(\mathbb{Z} / p \mathbb{Z})^{2}}\left(b^{2}+c^{2} \equiv-\bar{i} a \bmod p\right)\right),
$$

where $\bar{i}$ is the inverse of $i$ modulo $p$. Moreover, for any $a \in(\mathbb{Z} / p \mathbb{Z})^{*}$, setting $e(x)=$ $\exp (2 i \pi x)$ as customary, we have 


$$
\begin{aligned}
\left|\left\{(b, c) \in(\mathbb{Z} / p \mathbb{Z})^{2} \mid b^{2}+c^{2} \equiv a \bmod p\right\}\right| & =\sum_{(b, c) \in(\mathbb{Z} / p \mathbb{Z})^{2}} \frac{1}{p} \sum_{h \in \mathbb{Z} / p \mathbb{Z}} e\left(\frac{h\left(b^{2}+c^{2}-a\right)}{p}\right) \\
& =\frac{1}{p}\left(\sum_{h \in(\mathbb{Z} / p \mathbb{Z})^{*}} e\left(-\frac{h a}{p}\right)\left(\sum_{b \in \mathbb{Z} / p \mathbb{Z}} e\left(\frac{h b^{2}}{p}\right)\right)^{2}+p^{2}\right) \\
& = \begin{cases}p-1 & \text { if } p \equiv 1 \bmod 4 \\
p+1 & \text { if } p \equiv-1 \bmod 4 \\
p & \text { if } p=2 .\end{cases}
\end{aligned}
$$

The last equality follows from the classical computation of Gauss sums (see [11, 3.38]). For $p \geq k$, this leads to

$$
\beta_{p}= \begin{cases}\left(1+\frac{1}{p-1}\right)^{k}\left(1-\frac{k}{p}+2 \frac{k-1}{p^{2}}-\frac{k-1}{p^{3}}\right) & \text { if } p \equiv 1 \bmod 4 \\ \left(1+\frac{1}{p-1}\right)^{k}\left(1-\frac{k}{p}+\frac{k-1}{p^{3}}\right) & \text { if } p \equiv-1 \bmod 4\end{cases}
$$

It is easy to compute the local factors for $p \leq k$. We find that

$$
\beta_{p}= \begin{cases}\left(\frac{p}{p-1}\right)^{k} \frac{(p-1)(2 p-1)}{p^{3}} & \text { if } p \equiv 1 \bmod 4 \\ \left(\frac{p}{p-1}\right)^{k} \frac{p-1}{p^{3}} & \text { if } p \equiv-1 \bmod 4 \\ 2^{k-2} & \text { if } p=2 .\end{cases}
$$

We notice that $\beta_{p}$ is nonzero for every $p$ and that $\beta_{p}=1+O\left(p^{-2}\right)$, thus $\prod_{p} \beta_{p}$ is a nonzero convergent product. We prove in Lemma A.3 that the product of the local factors is always convergent for systems of finite complexity.

Corollary 1.3 counts the number of weighted arithmetic progressions of primes up to $N$ whose common difference is a sum of two squares, each such arithmetic progression being weighted by the number of representation of the common difference. To count these progressions without multiplicity, one has to replace $R$ by the indicator function $1_{S}$ of the sums of two squares. The very recent work of Matthiesen on multiplicative functions [14] shows how to deal with $1_{S}$ in linear averages, so that the count without multiplicity can be derived along the same lines as the count with multiplicity. We refrain from doing it here for brevity, but we note that this differs from the results of Tao and Ziegler [19], which count necessarily multiplicities.

In general, the only polynomial patterns we are able to deal with are the ones which can be converted into linear patterns by the use of representation functions of PDBQFs, as in the proof of Corollary 1.3. The ability to deal with arithmetic progressions whose common difference is a sum of two squares as if they were a linear pattern is reminiscent of a result of Green [6]: he proved that if a set $A \subset[N]$ does not contain any such progression of length 3 , then $|A| \ll N(\log \log N)^{-c}$ for some $c>0$. 
1.4. Tuples of primes whose pairwise midpoints are sums of two squares. Theorem 1.2 can yield many further asymptotics for the number of solutions to equations in primes and sums of squares, some of which are not covered by Tao and Ziegler [19]. This is the case of the equation $p_{1}+p_{2}=n_{1}^{2}+n_{2}^{2}$ with $p_{1}, p_{2}$ primes and $n_{1}, n_{2}$ integers. The underlying polynomial system is

$$
\left(n_{1}, n_{2}, n_{3}\right) \mapsto\left(n_{1}, n_{2}^{2}+n_{3}^{2}-n_{1}\right)
$$

which is not of the form $\left(n_{1}, n_{1}+P(\mathbf{r})\right)$, hence not a polynomial progression. More generally, by analogy to a theorem of Balog [1], we consider tuples of odd primes $p_{1}, \ldots, p_{d}$ such that $\left(p_{i}+p_{j}\right) / 2$ is a sum of two squares for all $i \neq j$. To determine the asymptotic for such tuples, we analyse the sum

$$
\sum_{1 \leq n_{1}, \ldots, n_{d} \leq N} \prod_{i \in[d]} \Lambda\left(2 n_{i}+1\right) \prod_{j \neq k} R\left(n_{j}+n_{k}+1\right)
$$

where $R$ is again the representation function of sums of two squares. The system of linear forms at hand is of finite complexity, so that Theorem 1.2 applies.

1.5. Other results within the scope of our method. We claim, but we do not formally prove, that our method yields a result similar to Theorem 1.2 with the divisor function $\tau$ instead of the representation functions $R_{f_{i}}$. In fact, this result is easier to prove, since the treatment of the representation function of a binary quadratic form by Matthiesen [16] relies on her earlier paper on the divisor function [15].

Theorem 1.4. Let $\Psi=\left(\psi_{1}, \ldots, \psi_{t+s}\right): \mathbb{Z}^{d} \rightarrow \mathbb{Z}^{t+s}$ be a system of affine-linear forms of finite complexity. Suppose that the coefficients of the linear part $\dot{\Psi}$ are bounded by L. Let $K \subset[-N, N]^{d}$ be a convex body such that $\Psi(K) \subset[0, N]^{t+s}$. Write $\Phi=\left(\psi_{t+1}, \ldots, \psi_{t+s}\right)$ and $\dot{\Phi}$ for the linear part. Then

$$
\sum_{n \in \mathbb{Z}^{d} \cap K} \prod_{i=1}^{t} \Lambda\left(\psi_{i}(n)\right) \prod_{j=t+1}^{t+s} \tau\left(\psi_{j}(n)\right)=(\log N)^{s} \beta_{\infty} \prod_{p} \beta_{p}+o_{d, t, s, L}\left(N^{d} \log ^{s} N\right)
$$

where

$$
\beta_{\infty}=\operatorname{Vol}(K)
$$

and

$$
\beta_{p}=\left(\frac{p}{p-1}\right)^{t-s} \mathbb{E}_{a \in[p]^{d}} \prod_{i=1}^{t} 1_{\left(\psi_{i}(a), p\right)=1} \sum_{\left(k_{1}, \ldots, k_{s}\right) \in \mathbb{N}^{s}} \alpha_{\Phi_{a, p}}\left(p^{k_{1}}, \ldots, p^{k_{s}}\right)
$$

with $\Phi_{a, p}: b \mapsto \Phi(a)+p \dot{\Phi}(b)$ and $\alpha$ as in Definition $A .1$.

This theorem provides an asymptotic for the number of triples of nonnegative integers $(a, b, c)$ such that $a, a+b c, a+2 b c$ are primes. This is again a quadratic pattern; in fact, $\tau$ can be viewed as the representation function of the quadratic form $(x, y) \mapsto x y$. We can obtain a result similar to Corollary 1.3. We let

$$
L=\left\{( a , b , c ) \in \left[1,+\infty\left[{ }^{3} \mid a+(k-1) b c \leq N\right\} .\right.\right.
$$


This is not a convex body, but we have $\operatorname{Vol}(L) \sim\left|L \cap \mathbb{Z}^{3}\right| \sim N^{2} \log N /(k-1)$. It is not difficult to deduce from Theorem 1.4 that

$$
\sum_{(a, b, c) \in L \cap \mathbb{Z}^{3}} \prod_{i=0}^{t-1} \Lambda(a+i b c)=\operatorname{Vol}(L) \prod_{p} \beta_{p}+o\left(N^{2} \log N\right)
$$

with

$$
\beta_{p}=\prod_{i=0}^{t-1} \Lambda_{p}(a+i b c)
$$

Again this result has the same shape as the Green-Tao theorem although the configuration involved is nonlinear.

We remark that the idea of mixing $\Lambda$ and $\tau$ is quite old. Titchmarsh [20] considered sums such as

$$
\sum_{p \leq N} \tau(p+a)
$$

or equivalently

$$
\sum_{n \leq N} \Lambda(n) \tau(n+a)
$$

for $a \in \mathbb{Z}$. Assuming the Riemann hypothesis, he proved that that

$$
\sum_{n \leq N} \Lambda(n) \tau(n+a)=c_{1}(a) x \log x+O(x \log \log x)
$$

for some explicit constant $c_{1}(a)$. The result was proven unconditionally by Linnik [13]. Fouvry [5] proved the refined asymptotic formula

$$
\sum_{n \leq N} \Lambda(n) \tau(n+a)=c_{1}(a) x \log x+C_{2}(a) \operatorname{Li}(x)+O_{A}\left(x(\log x)^{-A}\right)
$$

for any $A>0$. Notice that this problem does not belong to the scope of our method, because the involved linear system is of infinite complexity.

We also mention that Matthiesen, together with Browning [2], was able to generalise her result about quadratic forms to norm forms originating from a number field. This implies a generalisation of Theorem 1.2, but we refrain, for the sake of simplicity, from inspecting this general case.

1.6. Overview of the general strategy. We now turn to a proof of the main theorem, Theorem 1.2. The proof follows the usual Green-Tao method. In Section 2, we perform the $W$-trick to suppress the preference of the von Mangoldt function and the representation function for some residue classes. Because of the notably different behaviours of these functions with respect to arithmetic progressions, this is a delicate matter. Assuming some convergence properties of the local factors, which we prove in Appendix A, the 
implementation of the $W$-trick reduces the main theorem to Theorem 2.5, the statement that a multilinear average

$$
\mathbb{E}_{n \in \mathbb{Z}^{d} \cap K}\left(F_{0}\left(\psi_{0}(n)\right)-1\right) \prod_{i=1}^{t} F_{i}\left(\psi_{i}(t)\right)
$$

is asymptotically o(1). Thanks to a generalised von Neumann theorem, it suffices to ensure that $F_{0}-1$ has small Gowers uniformity norm and that all the functions $F_{i}$ and $F_{0}-1$ are bounded by a common enveloping sieve or pseudorandom majorant. This is where the novelty of our paper lies. While individual pseudorandom majorants for $\Lambda$ and for $R_{f}$ are known, we need to construct a common one that works for $\Lambda$ and $R_{f}$ simultaneously.

We state the von Neumann theorem and prove Theorem 2.5 in Section 3, assuming the majorant introduced then is sufficiently pseudorandom. The required pseudorandomness property is proven in Appendix B. Appendix C provides some general background around the notion of local density, i.e. the density of zeros of a linear system modulo a prime power.

\section{Proof of Theorem 1.2}

We fix some arbitrarily large integer $N$, so that our asymptotic results are valid in the limit where $N$ tends to infinity. We use the notation $[N]$ for the set of the first $N$ integers. Many of the parameters introduced in the sequel implicitly depend on $N$ (such as the convex body $K$, the map $p \mapsto \iota(p)$, the numbers $w, W, \bar{W}$, the set $\left.X_{0} \ldots\right)$.

2.1. Elimination of a negligible set. We start our proof by taking care of a technicality. We would like to eliminate slightly awkward integers from the support of the von Mangoldt and the representation functions. In fact, it will turn out handy to exclude prime powers and small primes from the support of $\Lambda$, so we introduce $\Lambda^{\prime}=1_{\mathcal{P} \backslash\left[N^{2 \gamma}\right]}$ log, for some constant $\gamma \in(0,1 / 2)$ to be fixed later. It coincides with $\Lambda$ on the bulk of its support up to $N$, namely large primes.

Similarly, there is a fairly sparse subset $X_{0} \subset[N]$, depending on some constants $C_{1}>0$ and $\gamma>0$, on which the divisor function, and also the representation function, behave abnormally, so that our process of majorising by a pseudorandom measure (carried out in Section 4) fails there. We recall the following definition originating from [15] and taken up in [16].

Definition 2.1. Let $\gamma=2^{-k}$ for some $k \in \mathbb{N}$ and let $C_{1}>1$. We define $X_{0}=X_{0}\left(\gamma, C_{1}, N\right)$ to be the set containing 0 and the set of positive integers $n \leq N$ satisfying either

(1) $n$ is excessively "rough", i.e. divisible by some large prime power $p^{a}>\log ^{C_{1}} N$ with $a \geq 2$, or

(2) $n$ is excessively smooth in the sense that if $n=\prod_{p} p^{a_{p}}$ then

$$
\prod_{p \leq N^{(1 / \log \log N)^{3}}} p^{a_{p}} \geq N^{\gamma / \log \log N}
$$

or 
(3) $n$ has a large square divisor $m^{2} \mid n$, which satisfies $m>N^{\gamma}$.

The following lemma, which is Lemma 3.2 from [16], itself a synthesis of Lemmas 3.2 and 3.3 from [15], shows how negligible this set is.

Lemma 2.1. For $\Psi$ and $K$ as in Theorem 1.2, we have

$$
\mathbb{E}_{n \in K \cap \mathbb{Z}^{d}} \sum_{i=t+1}^{t+s} 1_{\psi_{i}(n) \in X_{0}} \ll_{\gamma, d, s} \log ^{-C_{1} / 2} N .
$$

This enables us to state the next lemma, which allows us to ignore $X_{0}$ altogether. For any $\operatorname{PDBQF} f$, we use to the notation $\overline{R_{f}}(n)$ to denote $1_{n \notin X_{0}} R_{f}(n)$.

Lemma 2.2. If the parameter $C_{1}$ in Definition 2.1 is large enough, and for any choice of the constant $\gamma \in(0,1 / 2)$, Theorem 1.2 holds if and only if, under the same conditions, we have

$$
\sum_{n \in K \cap \mathbb{Z}^{d}} \prod_{i=1}^{t} \Lambda^{\prime}\left(\psi_{i}(n)\right) \prod_{j=t+1}^{t+s} \bar{R}_{f_{j}}\left(\psi_{j}(n)\right)=\beta_{\infty} \prod_{p} \beta_{p}+o\left(N^{d}\right) .
$$

Proof. We show first that

$$
\sum_{\substack{n \in K \cap \mathbb{Z}^{d} \\ \exists j \in \llbracket t+1 ; t+s \rrbracket: \psi_{j}(n) \in X_{0}}} \prod_{i=1}^{t} \Lambda\left(\psi_{i}(n)\right) \prod_{j=t+1}^{t+s} R_{f_{j}}\left(\psi_{j}(n)\right)=o\left(N^{d}\right) .
$$

Notice the use of the notation $\llbracket t+1 ; t+s \rrbracket=\{t+1, \ldots, t+s\}$. We get rid of the von Mangoldt factors by bounding their product by $\log ^{t} N$. Then we use the Cauchy-Schwarz inequality followed by the triangle inequality, which implies that

$$
\left(\sum_{\substack{n \in K \cap \mathbb{Z}^{d} \\ \exists j \in \llbracket t+1 ; t+s \rrbracket: \psi_{j}(n) \in X_{0}}} \prod_{j=t+1}^{t+s} R_{f_{j}}\left(\psi_{j}(n)\right)\right)^{2} \leq \sum_{n \in K \cap \mathbb{Z}^{d}}\left(\prod_{j=t+1}^{t+s} R_{f_{j}}\left(\psi_{j}(n)\right)\right)^{2} \sum_{n \in K \cap \mathbb{Z}^{d}} \sum_{j=t+1}^{t+s} 1_{\psi_{j}(n) \in X_{0}} .
$$

Finally, we use Lemma 3.1 of [16] which ensures that the first factor is $N^{d} \log ^{O_{s}(1)} N$ while the second is $N^{d} \log ^{-C_{1} / 2} N$ according to Lemma 2.1, so that taking $C_{1}$ larger than $2\left(t+O_{s}(1)\right)$, we have the result.

To replace $\Lambda$ by $\Lambda^{\prime}$, we remark that for each $i \in[t]$, the number of $n \in K \cap \mathbb{Z}^{d}$ such that $\psi_{i}(n) \leq N^{2 \gamma}$, resp. $\psi_{i}(n)$ is a prime power and not a prime, is $O\left(N^{d-1+2 \gamma}\right)$, resp. $O\left(N^{d-1} \log N \sqrt{N}\right)$. Using Cauchy-Schwarz or even pointwise bounds such as the divisor bound $R_{f_{j}}(n) \ll \tau(n) \ll_{\epsilon} N^{\epsilon}$, we conclude the proof of Lemma 2.2.

From now on, we will drop the bar, so that $R_{f}$ coincides with the actual representation function of $f$ on $[N] \backslash X_{0}$ and is 0 on $X_{0}$. 
2.2. Implementation of the $W$-trick. The $W$-trick is by now fairly standard; see [7], [8] for its implementations by Green and Tao, see also [15] and [16], which we are going to follow more closely. The idea is to eliminate the obvious bias of the primes, like the strong preference for odd numbers, to produce a more uniform set. The representation function of a PDBQF is also biased (it does not have the same average on every residue class), so this has to be corrected, too. To do this we introduce, for some slowly growing function of $N$, such as $w(N)=\log \log \log N$, the products

$$
W=\prod_{p \leq w} p \quad \text { and } \quad \bar{W}=\prod_{p \leq w} p^{\iota(p)},
$$

where $\iota(p)$ is defined by

$$
p^{\iota(p)-1}<\log ^{C_{1}+1} N \leq p^{\iota(p)}
$$

for some $C_{1}$ large enough as in Lemma 2.2. We observe that

$$
\left.\bar{W} \leq \prod_{p \leq w} p \log ^{C_{1}+1} N \ll \exp \left(\left(C_{1}+1\right) w \log \log N\right)\right)
$$

which is less than any power of $N$. In particular, we can decide that $\bar{W}<N^{\gamma}-1$ by choosing $N$ large enough.

Green and Tao did not need prime powers in their $W$, but in the case of a representation function of a PDBQF, they turn out to be necessary. Notice that for $N$ large enough, for $p \leq w(N)=\log \log \log N$, we always have $\iota(p) \geq 1$. We also introduce, for $b \in[\bar{W}]$, the function $\Lambda_{b, \bar{W}}^{\prime}$ defined by

$$
\Lambda_{b, \bar{W}}^{\prime}(n)=\frac{\phi(W)}{W} \Lambda^{\prime}(\bar{W} n+b),
$$

where $\Lambda^{\prime}=1_{\mathcal{P}} \log ($ we recall that $\phi(W) / W=\phi(\bar{W}) / \bar{W})$.

Unfortunately,

$$
\bar{W} \geq \exp \left(w(N) C_{1} \log \log N\right) \gg_{A} \log ^{A} N
$$

for any $A>0$, so it will not be possible, without a notable strengthening of the SiegelWalfisz theorem, to claim that $\Lambda_{b, \bar{W}}^{\prime}$ has average $1+o(1)$. A fortiori, it will not be possible to claim that $\Lambda_{b, \bar{W}}^{\prime}-1$ has the required uniformity property, in contrast to the normal $W$-trick. We will be able to make do without this uniformity result.

We perform the $W$-trick on $R_{f}$ as well, for any PDBQF $f$ of discriminant $D$. Following Matthiesen [16, Definition 7.2], we define

$$
r_{f, b}^{\prime}(m):=\frac{\sqrt{-D}}{2 \pi} \frac{\bar{W}}{\rho_{f, b}(\bar{W})} R_{f}(\bar{W} m+b),
$$

for any $b$ such that $\rho_{f, b}(\bar{W})>0.2$ By construction, $R_{f}(n)$ equals 0 in the case where $n \in X_{0}$, in particular in the case where $n \equiv 0 \bmod p^{\iota(p)}$ with $p \leq w(N)$. Hence, $r_{f, b}^{\prime}=0$

\footnotetext{
${ }^{2}$ Even if $\rho_{f, b}(\bar{W})=0$, we will use the notation $r_{f, b}^{\prime}(m)$ with the understanding that it means 0 .
} 
if $b \equiv 0 \bmod p^{\iota(p)}$, so $r_{f, b}^{\prime}$ has the desired property. In fact (see [16, Definition 7.2]) for $b \neq 0 \bmod p^{\iota(p)}$ and any $p \leq w(N)$ satisfying $\rho_{f, b}(\bar{W})=0$, we have

$$
\mathbb{E}_{n \leq M} r_{f, b}^{\prime}(n)=1+O\left(\bar{W}^{3} M^{-1 / 2}\right) .
$$

This average in arithmetic progressions relies on elementary convex geometry and is valid uniformly in the modulus, in sharp contrast with the analogous result for primes.

We now decompose the left-hand side of (6) into sums over congruence classes. We write

$$
\mathbb{Z}^{d} \cap K=\bigcup_{a \in[\bar{W}]^{d}} \mathbb{Z}^{d} \cap K_{a}
$$

where

$$
K_{a}=\left\{x \in \mathbb{R}^{d} \mid \bar{W} x+a \in K\right\}
$$

is again a convex body. Putting

$$
F(n)=\prod_{i=1}^{t} \Lambda\left(\psi_{i}(n)\right) \prod_{j=t+1}^{t+s} R_{f_{j}}\left(\psi_{j}(n)\right),
$$

we can write the left-hand side of (6) as

$$
\sum_{n \in \mathbb{Z}^{d} \cap K} F(n)=\sum_{a \in[\bar{W}]^{d}} \sum_{n \in \mathbb{Z}^{d} \cap K_{a}} F(\bar{W} n+a) .
$$

Moreover, for $j \in[t+s]$, we can write $\psi_{j}(\bar{W} n+a)=\bar{W} \tilde{\psi}_{j}(n)+c_{j}(a)$ where $c_{j}(a) \in[\bar{W}]$ and $\tilde{\psi}_{j}$ is an affine-linear form differing from $\psi_{j}$ only in the constant term. We remark that if $\psi_{i}(a)$ is not coprime to $W$ for $i \in[t]$ or if $\rho_{f_{j}, \psi_{j}(a)}(\bar{W})=0$ or $\psi_{j}(a) \equiv 0 \bmod p^{\iota(p)}$ for some $j \in \llbracket t+1 ; t+s \rrbracket$ and some prime $p \leq w(N)$, then for each $n \in K_{a} \cap \mathbb{Z}^{d}$ we have $F(n)=03$. Thus the residues $a$ which bring a nonzero contribution to the right-hand side of (10) are all mapped by $\Psi$ to tuples $\left(b_{1}, \ldots, b_{t+s}\right)$ belonging to the following set.

Definition 2.2. We denote by $B_{t, s}$ the set of residues $b \in[\bar{W}]^{t+s}$ such that

(1) for any $i \in[t],\left(b_{i}, W\right)=1$;

(2) for any $j \in \llbracket t+1 ; t+s \rrbracket$ and any prime $p \leq w(N)$, we have $b_{j} \neq 0 \bmod p^{\iota(p)}$;

(3) for any $j \in \llbracket t+1 ; t+s \rrbracket, b_{j}$ is representable by $f_{j}$ modulo $\bar{W}$, that is, $\rho_{f_{j}, b_{j}}(\bar{W})>0$. Moreover, for an affine-linear system $\Psi: \mathbb{Z}^{d} \rightarrow \mathbb{Z}^{t+s}$, we define $A_{\Psi}$ to be the set of all $a \in[W]^{d}$ such that $\left(c_{i}(a)\right)_{i \in[t+s]} \in B_{t, s}$. We recall that $c_{i}(a)$ is the reduction modulo $\bar{W}$ in $[\bar{W}]$ of $\psi_{i}(a)$; we will also denote by $c(a)$ the vector $\left(c_{i}(a)\right)_{i \in[t+s]}$. We usually drop the subscripts on $B_{t, s}$ and $A_{\Psi}$ when no ambiguity is possible.

\footnotetext{
${ }^{3}$ Even if $\left(\psi_{i}(a), W\right)>1$, the integer $\psi_{i}(a)$ could still be a prime $p \leq w(N)<N^{\gamma}$, but given that primes smaller than $N^{\gamma}$ are not in the support of $\Lambda^{\prime}$, we still have $F(n)=0$.
} 
Now we rewrite (10) as

$$
\begin{aligned}
\sum_{n \in \mathbb{Z}^{d} \cap K} F(n) & =\sum_{a \in A_{\Psi}}\left(\frac{W}{\phi(W)}\right)^{t} \prod_{j=t+1}^{t+s} \frac{2 \pi}{\sqrt{-D_{j}}} \frac{\rho_{f_{j}, \psi_{j}\left(a_{j}\right)}(\bar{W})}{\bar{W}} \sum_{n \in K_{a} \cap \mathbb{Z}^{d}} F_{a}^{\prime}(n) \\
& =\prod_{j=t+1}^{t+s} \frac{2 \pi}{\sqrt{-D_{j}}} \sum_{a \in[\bar{W}]^{d}} Q(a) \sum_{n \in K_{a} \cap \mathbb{Z}^{d}} F_{a}^{\prime}(n),
\end{aligned}
$$

where

$$
Q(a)=\prod_{i=1}^{t} \Lambda_{W}\left(\psi_{i}(a)\right) \prod_{j=t+1}^{t+s} \frac{\rho_{f_{j}, \psi_{j}(a)}(\bar{W})}{\bar{W}} 1_{\forall p \leq w, \psi_{j}(a) \neq 0 \text { mod } p^{\iota(p)}},
$$

and

$$
F_{a}^{\prime}(n)=\prod_{i=1}^{t} \Lambda_{c_{i}(a), \bar{W}}^{\prime}\left(\tilde{\psi}_{i}(n)\right) \prod_{j=t+1}^{t+s} r_{f_{j}, c_{j}(a)}^{\prime}\left(\tilde{\psi}_{j}(n)\right) .
$$

Furthermore, we use the identity

$$
F_{a}^{\prime}(n)=\prod_{i=1}^{t} \Lambda_{c_{i}(a), \bar{W}}^{\prime}\left(\tilde{\psi}_{i}(n)\right)+\sum_{j=1+t}^{t+s}\left(r_{f_{j}, c_{j}(a)}^{\prime}\left(\tilde{\psi}_{j}(n)\right)-1\right) F_{a, j}^{\prime}(n),
$$

where

$$
F_{a, j}^{\prime}(n)=\prod_{k<j} r_{f_{k}, c_{k}(a)}^{\prime}\left(\tilde{\psi}_{k}(n)\right) \prod_{i=1}^{t} \Lambda_{c_{i}(a), \bar{W}}^{\prime}\left(\tilde{\psi}_{i}(n)\right) .
$$

Thus, equation (10) yields

$$
\left(\prod_{j=t+1}^{t+s} \frac{2 \pi}{\sqrt{-D_{j}}}\right)^{-1} \sum_{n \in \mathbb{Z}^{d} \cap K} F(n)=T_{1}+T_{2},
$$

where

$$
T_{1}=\sum_{a \in[\bar{W}]^{d}} Q(a) \sum_{n \in \mathbb{Z}^{d} \cap K_{a}} \prod_{i=1}^{t} \Lambda_{c_{i}(a), \bar{W}}^{\prime}\left(\tilde{\psi}_{i}(n)\right)
$$

and

$$
T_{2}=\sum_{j=t+1}^{t+s} \sum_{a \in A} \sum_{n \in \mathbb{Z}^{d} \cap K_{a}}\left(r_{f_{j}, c_{j}(a)}^{\prime}\left(\tilde{\psi}_{j}(n)\right)-1\right) F_{a, j}^{\prime}(n) .
$$

Here, the first term is expected to be the main term, of the order of magnitude of $\operatorname{Vol}(K)$, while the second one involving the difference of a $\bar{W}$-tricked representation function to its average 1 , is expected to be negligible, that is, $o\left(N^{d}\right)$. 
2.3. Analysis of the main term. To deal with the main term (13), ideally, we would like to claim that the inner sum satisfies

$$
\sum_{n \in \mathbb{Z}^{d} \cap K_{a}} \prod_{i=1}^{t} \Lambda_{c_{i}(a), \bar{W}}^{\prime}\left(\tilde{\psi}_{i}(n)\right)=\operatorname{Vol}\left(K_{a}\right)+o\left((N / \bar{W})^{d}\right) .
$$

Unfortunately, this statement, proven by Green and Tao [ $\underline{8}$, Theorem 5.1] with $W$ instead of $\bar{W}$, is beyond reach at the moment, basically because $\bar{W}$ is too large for the Siegel-Walfisz theorem to apply. If we were able to lower the prime powers $p^{\iota(p)} \approx \log ^{C_{1}} N$ involved in $\bar{W}$ to smaller prime powers $p^{\eta(p)} \approx \log \log N$, the resulting $\widetilde{W}$ would be small enough for Siegel-Walfisz (and more generally [8, Theorem 5.1]) to apply. Let us then define $\eta(p)$ by

$$
p^{\eta(p)-1}<\log \log N \leq p^{\eta(p)}
$$

and $\widetilde{W}=\prod_{p \leq w} p^{\eta(p)} \leq \prod_{p \leq w} p \log \log N \ll \exp \left((\log \log \log N)^{2}\right)$.

The reader may wonder at this point why we performed the $\bar{W}$ trick at all, if we really would like to deal with congruence classes modulo $\widetilde{W}$. The reason for this is that Lemma 2.2 would not hold if $X_{0}$ contained all numbers smaller than $N$ that have a prime power factor larger than $\log \log N$ : this is not a sparse enough set, given the possibly large values of $R_{f}$ and $\Lambda$. Thus, performing the $\widetilde{W}$-trick, we could not force the residues to satisfy $c_{j}(a) \neq 0 \bmod p^{\eta(p)}$, whereas the $\bar{W}$-trick allowed us to force $c_{j}(a) \neq 0 \bmod p^{\iota(p)}$. Imposing such a nonzero congruence will prove crucial to ensure that $r_{f_{j}, c_{j}(a)}^{\prime}$ is dominated by a pseudorandom majorant, and thus to ensure the term $T_{2}$ is negligible.

To lower the prime powers, we shall rely on the powerful lift-invariance property of Matthiesen [16. Lemma 6.3].

Lemma 2.3. Let $f$ be a PDBQF of discriminant $D$. Let $p_{0}$ be a prime and $\alpha \geq v_{p_{0}}(d)$ be an integer. Suppose $b \neq 0 \bmod p_{0}^{\alpha}$. Then for all $\beta \geq \alpha$ and $c \equiv b \bmod p_{0}^{\alpha}$, we have

$$
\rho_{f, b}\left(p_{0}^{\alpha}\right) p_{0}^{-\alpha}=\rho_{f, c}\left(p_{0}^{\beta}\right) p_{0}^{-\beta}
$$

To reduce prime powers, we decompose the residue set $[\bar{W}]^{d}$ into $X_{1}$ and $X_{2}$, where

$$
X_{1}=\left\{a \in[\bar{W}]^{d} \mid \forall j \in \llbracket t+1 ; t+s \rrbracket \quad \forall p \leq w(N) \quad \psi_{j}(a) \neq 0 \bmod p^{\eta(p)}\right\}
$$

and $X_{2}$ is the complement of $X_{1}$ in $[\bar{W}]^{d}$. We also introduce

$$
Y_{1}=\left\{a \in[\widetilde{W}]^{d} \mid \forall j \in \llbracket t+1 ; t+s \rrbracket \quad \forall p \leq w(N) \quad \psi_{j}(a) \neq 0 \bmod p^{\eta(p)}\right\} .
$$

First, for $a \in X_{1}$, we remark that $Q(a)$ depends only on the reduction $\tilde{a} \in Y_{1}$ of $a$. Indeed, writing

$$
\widetilde{Q}(a)=\prod_{i=1}^{t} \Lambda_{W}\left(\psi_{i}(a)\right) \prod_{j=t+1}^{t+s} \frac{\rho_{f_{j}, \psi_{j}(a)}(\widetilde{W})}{\widetilde{W}} 1_{\forall p \leq w, \psi_{j}(a) \neq 0 \bmod p^{\eta(p)}}
$$


we have $\widetilde{Q}(\tilde{a})=Q(a)$. This shows that

$$
\begin{aligned}
\sum_{a \in X_{1}} Q(a) \sum_{n \in \mathbb{Z}^{d} \cap K_{a}} \prod_{i=1}^{t} \Lambda_{c_{i}(a), \bar{W}}^{\prime}\left(\tilde{\psi}_{i}(n)\right) & =\sum_{a \in Y_{1}} Q(a) \sum_{\substack{b \in[\bar{W}]^{d} \\
b \equiv a \bmod \widetilde{W}^{\prime}}} \sum_{n \in \mathbb{Z}^{d} \cap K_{b}} \prod_{i=1}^{t} \Lambda_{c_{i}(b), \bar{W}}^{\prime}\left(\tilde{\psi}_{i}(n)\right) \\
& =\sum_{a \in Y_{1}} Q(a) \sum_{n \in \mathbb{Z}^{d} \cap K_{a}} \prod_{i=1}^{t} \Lambda_{c_{i}(a), \widetilde{W}}^{\prime}\left(\tilde{\psi}_{i}(n)\right) .
\end{aligned}
$$

We admit a slight abuse of notation: in the last term, $\tilde{\psi}_{i}$ may be different from the other occurrences of $\tilde{\psi}_{i}$ (differing at most in the constant term) and $c_{i}(a) \equiv \psi_{i}(a) \bmod \widetilde{W}$ lies in $[\widetilde{W}]$. They satisfy $\psi_{i}(\widetilde{W} n+a)=\widetilde{W} \tilde{\psi}_{i}(n)+c_{i}(a)$. Now we claim that an asymptotic for the inner sum follows from the work of Green and Tao [8]. To check this, notice that the properties of $W=W(N)$ that are used there to prove Theorem 5.1 are the following.

- There is a function $w(N)$ tending to infinity such that every prime $p \leq w(N)$ divides $W$. This is still the case for $\widetilde{W}$, as one can easily check that $\eta(p) \geq 1$ for all $p \leq \log \log \log N$.

- The exceptional primes for the system $\widetilde{\Psi}$, that is primes $p$ modulo which two forms of the systems are affinely dependent, are $O(w)=O(\log \log N)$; this is still true in our setting. This bound was important in the application of Theorem D.3 to prove Proposition 6.4 in [8].

- The size of $W$ is reasonable, namely $W=O(\log N)$; this is crucial to derive equation (12.9) from equation (12.10) in [8]. We also have this bound for $\widetilde{W}$.

Thus for any $a \in Y_{1}$ that has a nonzero contribution, in particular, satisfying $\left(c_{i}(a), W\right)=1$ for all $i \in[t]$, we get

$$
\sum_{n \in \mathbb{Z}^{d} \cap K_{a}} \prod_{i=1}^{t} \Lambda_{c_{i}(a), \widetilde{W}}^{\prime}\left(\tilde{\psi}_{i}(n)\right)=\operatorname{Vol}\left(K_{a}\right)+o\left((N / \widetilde{W})^{d}\right) .
$$

Inserting this equality in (16) yields

$$
\sum_{a \in X_{1}} Q(a) \sum_{n \in \mathbb{Z}^{d} \cap K_{a}} \prod_{i=1}^{t} \Lambda_{c_{i}(a), \bar{W}}^{\prime}\left(\tilde{\psi}_{i}(n)\right)=\left(\operatorname{Vol}(K)+o\left(N^{d}\right)\right) \mathbb{E}_{a \in[\widetilde{W}]^{d}} Q(a) 1_{a \in Y_{1}} .
$$

We exploit multiplicativity to write

$$
\mathbb{E}_{a \in[\widetilde{W}]^{d}} Q(a) 1_{a \in Y_{1}}=\prod_{p \leq w} \mathbb{E}_{a \in\left(\mathbb{Z} / p^{\eta(p)} \mathbb{Z}\right)^{d}} \prod_{i=1}^{t} \Lambda_{p}\left(\psi_{i}(a)\right) \prod_{j=t+1}^{t+s} \frac{\rho_{f_{j}, \psi_{j}(a)}\left(p^{\eta(p)}\right)}{p^{\eta(p)}} 1_{\psi_{j}(a) \neq 0 \text { mod } p^{\eta(p)}} .
$$

Now we invoke results from the Appendix A to conclude. Indeed, replacing $\iota(p)$ by $\eta(p)$ in Lemma A.2, we find that

$$
\mathbb{E}_{a \in\left(\mathbb{Z} / p^{\eta(p)} \mathbb{Z}\right)^{d}} \prod_{i=1}^{t} \Lambda_{p}\left(\psi_{i}(a)\right) \prod_{j=t+1}^{t+s} \frac{\rho_{f_{j}, \psi_{j}(a)}\left(p^{\eta(p)}\right)}{p^{\eta(p)}} 1_{\psi_{j}(a) \neq 0 \bmod p^{\eta(p)}}=\beta_{p}+O\left((\log \log N)^{-1 / 3}\right) .
$$


Using Lemma A.3, we conclude that

$$
\mathbb{E}_{a \in[\widetilde{W}]^{d}} Q(a) 1_{a \in Y_{1}}=\prod_{p} \beta_{p}+o(1)
$$

and finally we can write

$$
\sum_{a \in X_{1}} Q(a) \sum_{n \in \mathbb{Z}^{d} \cap K_{a}} \prod_{i=1}^{t} \Lambda_{c_{i}(a), \bar{W}}^{\prime}\left(\tilde{\psi}_{i}(n)\right)=\beta_{\infty} \prod_{p} \beta_{p}+o\left(N^{d}\right) .
$$

2.4. The sum over $X_{2}$. We now turn to the sum over $X_{2}$, which we would like to show is $o\left(N^{d}\right)$. Lacking an asymptotic for the inner sum, we shall be content with an upper bound. Luckily, such a bound is available, thanks to a majorant of the von Mangoldt function devised by Goldston and Yıldırım; see [8, Appendix D] and the references therein. Let us introduce

$$
\Lambda_{\chi, R}(n)=\log R\left(\sum_{\ell \mid n} \mu(\ell) \chi\left(\frac{\log \ell}{\log R}\right)\right)^{2},
$$

where $R=N^{\gamma}$ and $\gamma$ is to be chosen later, and $\chi$ is a smooth even function $\mathbb{R} \rightarrow[0,1]$ supported on $[-1,1]$ satisfying $\chi(0)=1$ and $\int_{0}^{1} \chi^{\prime}(x)^{2} d x=1$. Finally, for any $b \in[\bar{W}]$ coprime to $\bar{W}$, we define the Green-Tao majorant

$$
\nu_{\mathrm{GT}, b}: n \mapsto \frac{\phi(W)}{W} \Lambda_{\chi, R}(\bar{W} n+b)
$$

The following lemma shows that this function majorises the $\bar{W}$-tricked von Mangoldt function.

Lemma 2.4. For any $b \in[\bar{W}]$ coprime to $W$, we have

$$
\Lambda_{b, \bar{W}}^{\prime}(n) \ll \nu_{\mathrm{GT}, b}(n)
$$

for $n \in\left[R, N^{\prime}\right]$, where the implied constant depends only on $\gamma$.

Proof. To prove the first part of the lemma, we have to take care only of the integers $n \in\left[R, N^{\prime}\right]$ such that $\bar{W} n+b$ is prime. In this case, the left-hand side is bounded above by a constant multiple of $\frac{\phi(W)}{W} \log N$ while the right-hand side is $\frac{\phi(W)}{W} \log R$, but $\log R=$ $\gamma \log N \gg \log N$.

Notice that the bound is in fact valid on $\left[N^{\prime}\right]$, because if $n \leq R=N^{\gamma}$, we obviously have $\bar{W} n+b \leq N^{\gamma}(\bar{W}+1)<N^{2 \gamma}$ because $N$ is large enough, and $\Lambda^{\prime}(\bar{W} n+b)=0$ by the definition of $\Lambda^{\prime}$ (see Subsection 2.1).

Now if $\gamma$ is small enough, $\nu_{\mathrm{GT}, b}$ is known to satisfy the linear forms condition. This was shown by Green and Tao (see [8, Appendix D] and the Appendix C of this paper) with $W$ instead of $\bar{W}$, but the reader may check that in this portion of their article, the bound 
$W=O(\log N)$ on the size of $W$ plays no role, so that the argument works just as well with $\bar{W}$. In particular,

$$
\sum_{n \in \mathbb{Z}^{d} \cap K_{a}} \prod_{i=1}^{t} \Lambda_{c_{i}(a), \bar{W}}^{\prime}\left(\tilde{\psi}_{i}(n)\right) \ll \sum_{n \in \mathbb{Z}^{d} \cap K_{a}} \prod_{i=1}^{t} \nu_{\mathrm{GT}, c_{i}(a)}\left(\tilde{\psi}_{i}(n)\right)=\operatorname{Vol}\left(K_{a}\right)+o\left((N / \bar{W})^{d}\right)
$$

Here we used crucially the fact that no two of the forms $\tilde{\psi}_{i}$ are rational multiple of one another; this follows from the finite complexity assumption on the original system $\Psi$. From this, we infer that

$$
\sum_{a \in X_{2}} Q(a) \sum_{n \in \mathbb{Z}^{d} \cap K_{a}} \prod_{i=1}^{t} \Lambda_{c_{i}(a), \bar{W}}^{\prime}\left(\tilde{\psi}_{i}(n)\right) \ll N^{d} \mathbb{E}_{a \in[\bar{W}]^{d}} Q(a) 1_{a \in X_{2}} .
$$

We use the triangle inequality to bound the inner expectation by

$$
\sum_{\substack{p \leq w \\ j \in \llbracket t+1 ; t+s \rrbracket}} \mathbb{E}_{a \in[\bar{W}]^{d}} 1_{p^{\eta(p)} \mid \psi_{j}(a)} Q(a),
$$

which, by multiplicativity, can be rewritten as

$$
\sum_{\substack{q \leq w \\ j \in \llbracket t+1 ; t+s \rrbracket}} \mathbb{E}_{a \in\left(\mathbb{Z} / q^{\iota(q)} \mathbb{Z}\right)^{d}} 1_{q^{\eta(q)} \mid \psi_{j}(a)} Q_{q}(a) \prod_{p \leq w, q \neq p} \mathbb{E}_{a \in\left(\mathbb{Z} / p^{\iota(p)} \mathbb{Z}\right)^{d}} Q_{p}(a) .
$$

Here, as the reader can guess, we introduced

$$
Q_{p}(a)=\prod_{i=1}^{t} \Lambda_{p}\left(\psi_{i}(a)\right) \prod_{j=t+1}^{t+s} \frac{\rho_{f_{j}, \psi_{j}\left(a_{j}\right)}\left(p^{\iota(p)}\right)}{p^{\iota(p)}} 1_{\psi_{j}(a) \neq 0 \bmod p^{\iota(p)}}
$$

for any prime $p$, so that $Q(a)=\prod_{p \leq w(N)} Q_{p}(a)$. Again, we invoke the results of Appendix A. Lemmas A.2 and A.3 imply that

$$
\prod_{p \leq w, q \neq p} \mathbb{E}_{a \in\left(\mathbb{Z} / p^{\iota(p)} \mathbb{Z}\right)^{d}} Q_{p}(a)=O(1)
$$

while the proof of Lemma A.1 shows that

$$
\mathbb{E}_{a \in\left(\mathbb{Z} / q^{\iota(q)} \mathbb{Z}\right)^{d}} 1_{q^{\eta(q)} \mid \psi_{j}(a)} Q_{q}(a)=O\left((\log \log N)^{-1 / 3}\right) .
$$

Because $w(N)=\log \log \log N$ is so small, we get as desired

$$
\sum_{a \in X_{2}} Q(a) \sum_{n \in \mathbb{Z}^{d} \cap K_{a}} \prod_{i=1}^{t} \Lambda_{c_{i}(a), \bar{W}}^{\prime}\left(\tilde{\psi}_{i}(n)\right)=o\left(N^{d}\right) .
$$


2.5. Reduction of the main theorem. Given the above discussion, the main theorem (Theorem 1.2) boils down to proving that the term $T_{2}$ defined in equation (14) is $o\left(N^{d}\right)$. This is a consequence of the next proposition.

Theorem 2.5. Let $d, t$ and $s$ be nonnegative integers, and let $f_{0}, f_{t+1}, \ldots, f_{t+s}$ be PDBQF. Let $N^{\prime}=N / \bar{W}$, and $\Phi=\left(\phi_{0}, \ldots, \phi_{1+t+s}\right)$ be a system of affine-linear forms $\mathbb{Z}^{d} \rightarrow \mathbb{Z}^{t+s+1}$ of finite complexity whose linear coefficients are bounded by a constant. Let $L \subset\left[0, N^{\prime}\right]^{d}$ be a convex set such that $\Phi(L) \subset\left[1, N^{\prime}\right]^{t+s+1}$. Then for any $b \in B_{t, s+1}$, we have

$$
\sum_{n \in \mathbb{Z}^{d} \cap L}\left(r_{f_{0}, b_{0}}^{\prime}\left(\phi_{0}(n)\right)-1\right) \prod_{i \in[t]} \Lambda_{b_{i}, \bar{W}}^{\prime}\left(\phi_{i}(n)\right) \prod_{j=t+1}^{t+s} r_{f_{j}, b_{j}}^{\prime}\left(\phi_{j}(n)\right)=o\left(N^{\prime d}\right) .
$$

The set $B=B_{t, s+1}$ was introduced in Definition 2.2. Notice the slight twist of notation with respect to the original definition, due to the fact that our quadratic forms are labelled $f_{0}, \ldots, f_{t+s+1}$.

We prove this theorem in the next section.

\section{Proof of Theorem 2.5}

3.1. Generalised von Neumann theorem and uniformity. Here and in the rest of the paper, $N^{\prime}=N / \bar{W}$. To prove Theorem $[2.5$, we have to show that the average along a linear system of a product is $o\left(N^{\prime d}\right)$, knowing that one of the factor has average $o\left(N^{\prime d}\right)$. To do so, we reduce to a family of standard linear systems, the ones which underlie the definition of Gowers norms which we now introduce.

Definition 3.1. Let $g: \mathbb{Z} \rightarrow \mathbb{R}$ be a function and $k \geq 1$ an integer. The Gowers norm or $U^{k}$ norm of $g$ on $[N]$ is the expression

$$
\|g\|_{U^{k}[N]}=\left(\mathbb{E}_{x \in[N]} \mathbb{E}_{h \in[N]^{k}} \prod_{\omega \in\{0,1\}^{k}} g(x+\omega \cdot h)\right)^{2^{-k}}
$$

We need one more definition.

Definition 3.2. Let $D \geq 1$. A D-pseudorandom measure is a sequence of functions $\nu=\nu_{M}: \mathbb{Z} / M \mathbb{Z} \rightarrow \mathbb{R}_{+}$, satisfying

(1) $\mathbb{E}_{n \leq M} \nu(n)=1+o(1)$.

(2) ( $D$-linear forms conditions) Let $1 \leq d, t \leq D$. For every finite-complexity system of affine-linear forms $\Psi: \mathbb{Z}^{d} \rightarrow \mathbb{Z}^{t}$ with coefficients bounded by $D$ and any convex set $K \subset[-M, M]^{d}$ such that $\Psi(K) \subset[M]^{t}$, the following estimate holds

$$
\mathbb{E}_{n \in \mathbb{Z}^{d} \cap K} \prod \nu\left(\psi_{i}(n)\right)=1+o(1) .
$$

\footnotetext{
${ }^{4}$ In earlier works such as [8] or [16], there was a correlation condition, but it is no longer necessary due to the work of Fox, Conlon and Zhao [3], and its integration by Tao and Ziegler [18].
} 
Pseudorandom measures are defined on cyclic groups rather than intervals of integers, so the values of the linear forms $\psi_{i}(n)$ are understood modulo $M$. Similarly, some authors prefer to define the Gowers norms on cyclic groups [8, Appendix B], and then in intervals of integers by embedding them in cyclic groups. However, the uniformity conditions and the von Neumann theorem will also work well with the definition above.

On the other hand, the functions we want to majorise, of the form $\Lambda_{b, \bar{W}}^{\prime}-1$ and $r_{f, b}^{\prime}-1$, are naturally defined on an interval $\left[N^{\prime}\right]$. When $N^{\prime} \leq M$ are integers and $f:\left[N^{\prime}\right] \rightarrow \mathbb{R}$ is a function, we embed $\left[N^{\prime}\right]$ in $\mathbb{Z} / M \mathbb{Z}$ canonically and extend the definition of $f$ to $\mathbb{Z} / M \mathbb{Z}$ by setting $f=0$ outside $\left[N^{\prime}\right]$. Passing from intervals to cyclic groups involves some technicalities. To avoid torsion and wrap-around issues, we try to embed an interval into a sufficiently large cyclic group of prime order

Following [8, Proposition 7.1], we can now state the generalised von Neumann theorem.

Theorem 3.1. Let $t, d, L$ be positive integer parameters. Then there are positive constants $1 \leq \Gamma$ and $D$, depending on $t, d$ and $L$ such that the following holds. Let $C$ be a constant satisfying $\Gamma \leq C \leq O_{t, d, L}(1)$ be arbitrary and suppose that $M \in\left[C N^{\prime}, 2 C N^{\prime}\right]$ is a prime. Let $\nu: \mathbb{Z} / M \mathbb{Z} \rightarrow \mathbb{R}^{+}$be a D-pseudorandom measure, and suppose that $f_{1}, \ldots, f_{t}:\left[N^{\prime}\right] \rightarrow \mathbb{R}$ are functions with $\left|f_{i}(x)\right| \leq \nu(x)$ for all $i \in[t]$ and $x \in\left[N^{\prime}\right]$. Suppose that $\Psi=\left(\psi_{1}, \ldots, \psi_{t}\right)$ is system of affine-linear forms of finite complexity whose linear coefficients are bounded by $L$. Let $K \subset[-M, M]^{d}$ be a convex set such that $\Psi(K) \subset[M]^{t}$. Finally, suppose that

$$
\min _{1 \leq j \leq t}\left\|f_{j}\right\|_{U^{t-1}[N]}=o(1) \text {. }
$$

Then we have

$$
\mathbb{E}_{n \in K \cap \mathbb{Z}^{d}} \prod_{i \in[t]} f_{i}\left(\psi_{i}(n)\right)=o(1)
$$

We highlight that this theorem actually replaces a linear system $\Psi$ with another one, the system $(x+\omega \cdot h)_{\omega \in\{0,1\}^{t-1}}$, so that it is not immediately obvious that we have reduced the difficulty. However, it happens that uniformity with respect to this system can be characterised in another way: this is the inverse theorem for the Gowers norms [10]. The following proposition provides the uniformity condition (19) for our functions.

Proposition 3.2. Let $f$ be a PDBQF, and $b \in[\bar{W}]$ be representable by $f$ modulo $\bar{W}$ and not divisible by any $p^{\iota(p)}$ for $p \leq w(N)$. Then the tricked representation function of $f$ defined by (9) satisfies

$$
\forall k \in \mathbb{N} \quad\left\|r_{f, b}^{\prime}-1\right\|_{U^{k}\left[N^{\prime}\right]}=o(1)
$$

The proof of this proposition [16, Sections 14-18] consists in evaluating the correlation of $r_{f, b}^{\prime}-1$ with nilsequences.

3.2. Construction of a pseudorandom majorant. We now construct a pseudorandom measure which dominates both the $\bar{W}$-tricked von Mangoldt and representation functions. 
3.2.1. The pseudorandom majorant of the von Mangoldt function. We first recall the pseudorandom majorant $\nu_{\mathrm{GT}, b}(n)$ from the Green-Tao machinery, first used by Goldston and Yıldırım. We already defined it in equation (17). Green and Tao [7, Lemma 9.7] proved that it has average $1+o(1)$.

Lemma 3.3. If $\gamma$ is small enough, for any $b \in[\bar{W}]$ coprime to $\bar{W}$, we have

$$
\mathbb{E}_{n \in\left[N^{\prime}\right]} \nu_{\mathrm{GT}, b}(n)=1+o(1) .
$$

3.2.2. The pseudorandom majorant of the representation function. We also use a pseudorandom majorant from Matthiesen's work. For this we need to recall some notation and facts from [16]. For a set $\mathcal{A}$ of primes, $\langle\mathcal{A}\rangle$ stands for the set of integers whose prime factors are all in $\mathcal{A}$, and $\tau_{D}(n)=\sum_{d \in\left\langle\mathcal{P}_{D}\right\rangle} 1_{d \mid n}$.

Proposition 3.4. For any integer $D \equiv 0,1 \bmod 4$, there exists a set of primes $\mathcal{P}_{D}$ of density 1/2, which is a union of congruence classes modulo $D$, such that putting $\mathcal{P}_{D}^{*}=$ $\mathcal{P}_{D} \cup\{p \in \mathcal{P}: p \mid D\}$ and $\mathcal{Q}_{D}=\mathcal{P} \backslash \mathcal{P}_{D}^{*}$, we have, for any PDBQF $f$ of discriminant $D$, the bound

$$
R_{f}(n) \ll_{D} \tau_{D}(n) \sum_{\substack{m \in\left\langle\mathcal{Q}_{D}\right\rangle \\ m^{2} \mid n}} 1_{\left\langle\mathcal{P}_{D}^{*}\right\rangle}\left(n / m^{2}\right) .
$$

To understand this result heuristically, which is the starting point of the construction of the pseudorandom majorant in [16], we recall that the number of representations of any odd number $n$ as a sum of two squares is $4 \sum_{d \mid n} \chi(d)$ where $\chi$ is the only nontrivial character modulo 4 . By multiplicativity, this is easily seen to become $\tau_{4}(n) \prod_{p \equiv 3 \bmod 4} 1_{v_{p}(n) \equiv 0 \bmod 2}$, with $\mathcal{P}_{4}$ being the set of primes congruent to 1 modulo 4 , from which we derive a majorant of the desired form. This works similarly for other quadratic forms.

Thus, to majorise the function $R_{f}$ it will be enough to majorise the functions $\tau_{D}$ and $1_{\left\langle\mathcal{P}_{D}^{*}\right\rangle}$. The heuristic to bound $\tau_{D}$ (or rather $\tau_{D} / \sqrt{\log N}$ ) is as follows (see [15, Lemma 4.1]). We would like to truncate the divisor sum defining it at $N^{\gamma}$ (possibly with a smooth cut-off), just as was done earlier for the von Mangoldt function. The function defined by this truncated divisor sum is called $\tau_{\gamma}$. Unfortunately, it turns out that the inequality $\tau \leq C \tau_{\gamma}$ is not entirely true, at least not true with the same constant $C$ throughout the first $N$ integers. Nevertheless, a heuristic of Erdős [4] says that an integer is either excessively rough or excessively smooth or has a cluster of many prime factors close together. Moreover we have excluded the first two possibilities when we took out the set $X_{0}$, so it remains to majorise $\tau(n)$ in the third case. Then the bound depends on the position of this cluster of primes and on its density. For more detail on the majorant of the divisor function, see [15.

To bound $1_{\left\langle\mathcal{P}_{D}^{*}\right\rangle}$ (or rather $1_{\left\langle\mathcal{P}_{D}^{*}\right\rangle} \sqrt{\log N}$ ), that is, the indicator function of the integers without any prime factor belonging to $\mathcal{Q}_{D}$, we use a sieving-type majorant, that is, a majorant similar to the one introduced above for the von Mangoldt function. Indeed, integers without any prime factor in $\mathcal{Q}_{D}$ are similar to prime numbers (integers without any non-trivial prime factor at all). 
To formalise this heuristic, let us introduce the following definition.

Definition 3.3. Let $\xi=2^{-m}$ for some $m \in \mathbb{N}$, put $\gamma=2 \xi$. We define sets $U(i, s)$ for integers $i, s$ as follows. For $i=\log _{2}(2 / \xi)-2=m-1$, we let $U(i, 2 / \xi)$ be $\{1\}$ and otherwise $U(i, 2 / \xi)=\emptyset$. If $s>2 / \xi$, write $U(i, s)$ for the set of all products of $m_{0}(i, s)=\left\lceil\xi s\left(i+3-\log _{2} s\right) / 100\right\rceil$ distinct primes from the interval $\left[N^{2^{-i-1}}, N^{2^{-i}}\right]$.

Let us fix an integer $D \equiv 0,1 \bmod 4$. We now propose a majorant for the $\mathrm{W}$-tricked representation function of a PDBQF of discriminant $D$, which was designed by Matthiesen [16]. We again need the smooth function $\chi$ (this should not be mistaken with a character, as there are no more characters in the sequel) introduced for the majorant of the von Mangoldt function. We use the function

$$
r_{D, \gamma}(n)=\frac{\beta_{D, \gamma}^{\prime}(n) \nu_{D, \gamma}^{\prime}(n)}{C_{D, \gamma}}
$$

where

$$
\nu_{D, \gamma}^{\prime}=\sum_{s=2 / \xi}^{(\log \log N)^{3}} \sum_{i=\log _{2} s-2} \sum_{u \in U(i, s)} 2^{s} 1_{u \mid n} \tau_{D, \gamma}^{\prime}(n),
$$

with

$$
\tau_{D, \gamma}^{\prime}(n)=\sum_{\substack{d \in\left\langle\mathcal{P}_{D}\right\rangle \\ p \mid d \Rightarrow p>w(N)}} 1_{d \mid n} \chi\left(\frac{\log d}{\log N^{\gamma}}\right)
$$

and

$$
\beta_{\gamma}^{\prime}(n)=\sum_{\substack{m \in\left\langle\mathcal{Q}_{D}\right\rangle \\ p \mid m \Rightarrow p>w(N) \\ m<N^{\gamma}}}\left(\sum_{\substack{e \in\left\langle\mathcal{Q}_{D}\right\rangle \\ p \mid e \Rightarrow p>w(N)}} 1_{m^{2} e \mid n} \mu(e) \chi\left(\frac{\log e}{\log N^{\gamma}}\right)\right)^{2} .
$$

The constant $C_{D, \gamma}$ is the one which ensures that the function $r_{D, \gamma}$ has average 1 ; the next lemma asserts the existence of such a constant.

We now define for any $b \in[\bar{W}]$ the function $\nu_{\text {Matt }, b, D}:\left[N^{\prime}\right] \rightarrow \mathbb{R}$ by

$$
\nu_{\mathrm{Matt}, b, D}(n)=r_{D, \gamma}(\bar{W} n+b) .
$$

The next lemma [16, Lemma 7.5] also asserts that this function is a pseudorandom majorant for the representation function of any PDBQF of discriminant $D$. Recall Definition 2.2.

Lemma 3.5. For any $P D B Q F f$ of discriminant $D$ and $b \in[\bar{W}]$ satisfying $b \neq 0 \bmod p^{\iota(p)}$ for any $p \leq w(N)$ and $\rho_{f, b}(\bar{W})>0$, the following bound holds

$$
r_{f, b}^{\prime}(n) \ll \nu_{\mathrm{Matt}, b, D}(n) .
$$

Furthermore, for some $C_{D, \gamma}=O(1)$, we have $\mathbb{E}_{n \in\left[N^{\prime}\right]} \nu_{\mathrm{Matt}, b, D}(n)=1+o(1)$. 
The crucial property of $\nu_{\text {Matt }}$ is that it is a truncated divisor sum, like $\nu_{\mathrm{GT}}$. Indeed, all divisors appearing are constrained to be less than $R=N^{\gamma}$. It is obvious by definition of $\chi$ for the divisors called $d, m, e$ and less obvious, but proven by Matthiesen, for $u$ (see Remark 3 following Proposition 4.2 in [16]). Moreover, the divisors $d, m, e$ only have prime factors larger than $w(N)$ (this feature is also present in $\Lambda_{\chi, R}$ ), while $u$ has only prime factors larger than $N^{(\log \log N)^{-3}}$.

3.2.3. Combination of both majorants. To be able to use the von Neumann theorem (Theorem 3.1), and thus establish Theorem 2.5, we need to bound all $t+s+1$ functions by the same majorant. Now each of them is bounded individually by some pseudorandom majorant defined above, so we define our common majorant by averaging all these majorants. Recall that $N^{\prime}=N / \bar{W}$; we take $M$ to be a prime satisfying $N^{\prime}<M \leq O\left(N^{\prime}\right)$. Given a family $f_{0}, f_{t+1}, \ldots, f_{t+s}$ of PDBQF of discriminants $D_{0}, D_{t+1}, \ldots, D_{t+s}$ and a family $\left(b_{0}, \ldots, b_{t+s}\right) \in B$, we define a function $\nu^{*}$ on $\left[N^{\prime}\right] \subset \mathbb{Z} / M \mathbb{Z}$ by

$$
\nu^{*}(n)=\frac{1}{t+s+2}\left(1+\sum_{i=1}^{t} \nu_{\mathrm{GT}, b_{i}}(n)+\sum_{j=t+1}^{t+s} \nu_{\mathrm{Matt}, b_{j}, D_{j}}(n)+\nu_{\mathrm{Matt}, b_{0}, D_{0}}(n)\right) .
$$

We extend it to $\mathbb{Z} / M \mathbb{Z}$ by setting $\nu^{*}(n)=1$ outside $\left[N^{\prime}\right]$. Our strategy of forming a common majorant for a family of functions by averaging a family of majorants is not really unheard of. In fact, Green and Tao [8] had to combine the majorants $n \mapsto \Lambda_{\chi, R}\left(\bar{W} n+b_{j}\right)$ for various $b_{j}$ and so did Matthiesen [16]. Notice also that Lê and Wolf [12] devised a certain condition of compatibility for two pseudorandom majorants. However, in our case the majorants have rather different origins. But they have a similar structure, the structure of a truncated divisor sum, so that the proof of the linear forms condition will not be much harder than the ones in [8] or [16].

We observe that $\nu^{*}$ satisfies

$$
1+\sum_{i=1}^{t} \Lambda_{b_{i}, \bar{W}}^{\prime}+\sum_{j=t+1}^{t+s} r_{f_{j}, b_{j}}^{\prime}+r_{f_{0}, b_{0}}^{\prime} \ll \nu^{*}
$$

and has average $1+o(1)$ by Lemmas 3.3 and 3.5. So to ensure that $\nu^{*}$ is a pseudorandom measure, it remains only to prove the linear forms condition (18). This is the content of the next proposition.

Proposition 3.6. Fix a constant $D>0$, and positive integers $t, s$. Then there exists a constant $C_{0}(D)$ such that the following holds. For any bounded $C \geq C_{0}(D)$ there exists $\gamma=\gamma(C, D)$ such that if $M \in\left[C N^{\prime}, 2 C N^{\prime}\right]$ is a prime, $b \in B_{t, s+1}$ and $f_{0}, f_{t+1}, \ldots, f_{t+s}$ are $P D B Q F$ and $\nu^{*}$ is defined as above, then $\nu^{*}$ satisfies the D-linear forms condition and for any $i \in[t]$ we have

$$
\Lambda_{\bar{W}, b_{i}}^{\prime} \ll \nu^{*} .
$$

Similarly, we have

$$
\left|r_{f_{0}, b_{0}}^{\prime}-1\right| \ll \nu^{*}
$$


and for any $j \in \llbracket t+1 ; t+s \rrbracket$, we have

$$
r_{f_{j}, b_{j}}^{\prime} \ll \nu^{*}
$$

where all inequalities are valid on $\left[N^{\prime}\right]$.

The inequalities have already been observed above. The linear forms condition will follow from the following proposition.

Proposition 3.7. Let $1 \leq d, t, s \leq D$, where $D$ is the constant appearing in Theorem 3.1. For any finite complexity system $\Psi: \mathbb{Z}^{d} \rightarrow \mathbb{Z}^{t+s}$ whose linear coefficients are bounded by $D$ and every convex $K \subset[0, N]^{d}$ such that $\Psi(K) \subset[1, N / \bar{W}]^{t}$, and any $b \in B$, the estimate

$$
\begin{array}{r}
\mathbb{E}_{n \in \mathbb{Z}^{d} \cap K} \prod_{j=t+1}^{t+s} r_{D_{j}, \gamma}\left(\bar{W} \psi_{j}(n)+b_{j}\right) \prod_{i \in[t]} \frac{\phi(W)}{W} \Lambda_{\chi, R}\left(\bar{W} \psi_{i}(n)+b_{i}\right) \\
=1+O_{D}\left(\frac{N^{d-1+O_{D}(\gamma)}}{\operatorname{Vol}(K)}\right)+o_{D}(1)
\end{array}
$$

holds, provided $\gamma$ is small enough.

Notice that the $t$ and $s$ are not the same as in Proposition 3.6. The proof is postponed to Appendix B.

Deriving the linear forms conditions for $\nu^{*}$ (Proposition 3.6) from Proposition 3.7 requires some extra work, because of the piecewise definition of $\nu^{*}$. This was done in 7 , Proposition 9.8] for instance, but see also [3, Proposition 8.4], where the same "localisation argument" is employed. Matthiesen also relies on it in [16]. The argument does not need any modification, so we do not reproduce it here and invite the reader to consult one of the references. We can now prove Theorem 2.5.

Proof of Theorem 2.5 assuming Proposition 3.6. Take any integers $d, t$ and $s$, and a system $\Phi: \mathbb{Z}^{d} \rightarrow \mathbb{Z}^{t+s+1}$ of affine-linear forms of finite complexity, where the coefficients of the linear part are bounded by $L$ and take $f_{0}, f_{t+1}, \ldots, f_{t+s}$ any PDBQF. Take a convex set $K \subset\left[1, N^{\prime}\right]^{d}$ such that $\Phi(K) \subset\left[N^{\prime}\right]^{t+s+1}$. Let $b \in B$. Then Proposition 3.1 and Proposition 3.6 provide constants $C_{0}$ and $\Gamma$, of which we take the maximum $C=\max \left(C_{0}, \Gamma\right)$. Now take a prime $M \in\left[C N^{\prime}, 2 C N^{\prime}\right]$. Such a prime exists by Bertrand's postulate. Define $\nu^{*}$ as above (22). Define $F_{0}=r_{f_{0}, b_{0}}^{\prime}-1$. Put $F_{i}=\Lambda_{b_{i}, \bar{W}}^{\prime}$ for $i \in[t]$ and $F_{j}=r_{f_{j}, b_{j}}^{\prime}$ for $j \in\{t+1, \ldots, t+s\}$. Then we have that $\left|F_{j}\right| \ll \nu^{*}$ for all $j \in\{0, \ldots, t+s\}$ and $\nu^{*}$ is a pseudorandom measure by Proposition 3.6, so that we can invoke the von Neumann theorem (Theorem 3.1). Together with the statements of Proposition 3.2 (specialised to $k=t+s)$, it implies Theorem 2.5.

We remark that although we want to prove a result concerning quadratic and not linear patterns in the primes, we do not need the polynomial forms condition introduced in [17]. This is because the polynomial character of our configurations is encapsulated in the representation functions of the quadratic forms. 
We have completed the proof our main theorem, conditionally on the following rather technical appendices. Appendix A provides estimates concerning the local factors that were used in Section 2. In Appendix B, we check the linear forms condition for the majorant introduced above, that is, we prove Proposition 3.6. Appendix $\mathrm{C}$ provides elementary justifications to some statements made in Appendices A and B.

\section{Appendix A. Analysis of the local faCtors $\beta_{p}$}

First, we check that the limit defining $\beta_{p}$ in Theorem 1.2 exists. We fix integers $d, t, s \geq 1$ and a system of linear forms $\Psi: \mathbb{Z}^{d} \rightarrow \mathbb{Z}^{t+s}$ of finite complexity, and we suppose its linear coefficients are bounded by $L$.

We also fix PDBQFs $f_{t+1}, \ldots, f_{t+s}$ of discriminants $D_{t+1}, \ldots, D_{t+s}$; these notions and the notation $\rho_{f_{j}}$ were defined in the introduction. Let $p$ be a fixed prime and $m \geq 1$ an integer. For $a \in\left(\mathbb{Z} / p^{m} \mathbb{Z}\right)^{d}$, let

$$
P_{m}(a)=\prod_{i=1}^{t} \Lambda_{p}\left(\psi_{i}(a)\right) \prod_{j=t+1}^{t+s} \frac{\rho_{f_{j}, \psi_{j}(a)}\left(p^{m}\right)}{p^{m}} .
$$

Finally, let $\beta_{p}(m)=\mathbb{E}_{a \in\left(\mathbb{Z} / p^{m} \mathbb{Z}\right)^{d}} P_{m}(a)$. Thus we want to prove that $\beta_{p}(m)$ is convergent as $m$ tends to $\infty$. This is a consequence of the following proposition

Proposition A.1. The sequence $\left(\beta_{p}(m)\right)_{m \in \mathbb{N}}$ is a Cauchy sequence. More precisely, there exists $M_{0}=M_{0}\left(D_{t+1}, \ldots, D_{t+s}\right)$ so that for all integers $m_{0} \geq M_{0}$ and $m, n \geq m_{0}$, we have

$$
\beta_{p}(m)-\beta_{p}(n)=O\left(m_{0}^{s} p^{-m_{0} / 2}\right)
$$

To facilitate the proof of this proposition and the further analysis of local factors, we ought to introduce a convenient notation present in both [8] and [16].

Definition A.1. For a given system of affine-linear forms $\Psi=\left(\psi_{1}, \ldots, \psi_{t}\right): \mathbb{Z}^{d} \rightarrow \mathbb{Z}^{t}$, positive integers $d_{1}, \ldots, d_{t}$ of lcm $m$, define the local divisor density by

$$
\alpha_{\Psi}\left(d_{1}, \ldots, d_{t}\right)=\mathbb{E}_{n \in(\mathbb{Z} / m \mathbb{Z})^{d}} \prod_{i=1}^{t} 1_{\psi_{i}(n) \equiv 0 \bmod d_{i}} .
$$

We now prove the proposition.

Proof. Let $M_{0}=\max _{j} v_{p}\left(D_{j}\right)$ and $m_{0} \geq M_{0}$. Let $m, n \geq m_{0}$. We split $\left(\mathbb{Z} / p^{m} \mathbb{Z}\right)^{d}$ into two parts

$$
A_{1}=A_{1}\left(m, m_{0}\right)=\left\{a \in\left(\mathbb{Z} / p^{m} \mathbb{Z}\right)^{d} \mid \forall j \in \llbracket t+1 ; t+s \rrbracket \quad \psi_{j}(a) \neq 0 \bmod p^{m_{0}}\right\}
$$

and

$$
A_{2}=A_{2}\left(m, m_{0}\right)=\left\{a \in\left(\mathbb{Z} / p^{m} \mathbb{Z}\right)^{d} \mid \exists j \in \llbracket t+1 ; t+s \rrbracket \quad \psi_{j}(a) \equiv 0 \bmod p^{m_{0}}\right\} .
$$

Thus

$$
\beta_{p}(m)=\mathbb{E}_{a \in\left(\mathbb{Z} / p^{m} \mathbb{Z}\right)^{d}} P(a) 1_{A_{1}\left(m, m_{0}\right)}(a)+\mathbb{E}_{a \in\left(\mathbb{Z} / p^{m} \mathbb{Z}\right)^{d}} P(a) 1_{A_{2}\left(m, m_{0}\right)}(a) .
$$


For the first term, we use the lift-invariance property [16, Corollary 6.4] already stated in Lemma 2.3. It implies that

$$
\mathbb{E}_{a \in\left(\mathbb{Z} / p^{m} \mathbb{Z}\right)^{d}} P(a) 1_{A_{1}\left(m, m_{0}\right)}=\mathbb{E}_{a \in\left(\mathbb{Z} / p^{m_{0}} \mathbb{Z}\right)^{d}} P(a) 1_{A_{1}\left(m_{0}, m_{0}\right)}
$$

thus the first term on the right-hand side of (25) does not depend on $m$. For the second term, we invoke the following general bound from [16] (see Lemma 6.3 and the proof of Lemma 8.2)

$$
\frac{\rho_{f_{j}, \psi_{j}(a)}\left(p^{m}\right)}{p^{m}} \ll \sum_{k=0}^{m} 1_{\psi_{j}(a) \equiv 0 \bmod p^{k}} .
$$

We also use the trivial bound $\Lambda_{p} \leq 2$ to infer the inequalities

$$
\begin{aligned}
P(a) 1_{A_{2}}(a) & \ll 2^{t} 1_{A_{2}}(a) \prod_{j=t+1}^{t+s} \sum_{k=0}^{m} 1_{\psi_{j}(a) \equiv 0 \bmod p^{k}} \\
& \ll m_{0}^{s} \sum_{\substack{0 \leq k_{t+1, \ldots, k_{t+s} \leq m} \\
\max k_{i} \geq m_{0}}} \prod_{j=t+1}^{t+s} 1_{\psi_{j}(a) \equiv 0 \bmod p^{k_{j}} .}
\end{aligned}
$$

Here the factor $m_{0}^{s}$ appears as the number of $s$-tuples whose entries are all in $\llbracket 0 ; m_{0}-1 \rrbracket$; moreover, the $2^{t}$ is merged with the implied constant, which crucially remains independent of $m$ or $m_{0}$. We then average over $a$ and let

$$
Z=\left(\zeta_{1}, \ldots, \zeta_{s}\right)=\left(\psi_{t+1}, \ldots, \psi_{t+s}\right)
$$

be the system of the $s$ last linear forms of $\Psi$, obtaining

$$
\mathbb{E}_{a \in\left(\mathbb{Z} / p^{m} \mathbb{Z}\right)^{d}} P(a) 1_{A_{2}}(a) \ll m_{0}^{s} \sum_{\substack{0 \leq k_{1}, \ldots, k_{s} \leq m \\ M:=\max k_{i} \leq m_{0}}} \mathbb{E}_{a \in\left(\mathbb{Z} / p^{M} \mathbb{Z}\right)^{d}} \prod_{i=1}^{s} 1_{p_{i}^{k} \mid \zeta_{i}} .
$$

We recognise the local density $\alpha_{Z}$ (see Definition A.1) on the right hand-side, so we put

$$
\delta_{p}=\sum_{\substack{0 \leq k_{1}, \ldots, k_{s} \leq m \\ M:=\max k_{i} \geq m_{0}}} \alpha_{Z}\left(p^{k_{1}}, \ldots, p^{k_{s}}\right),
$$

enabling us to rewrite (27) as

$$
\mathbb{E}_{a \in\left(\mathbb{Z} / p^{m} \mathbb{Z}\right)^{d}} P(a) 1_{A_{2}}(a) \ll m_{0}^{s} \delta_{p} .
$$

Since the linear coefficients of $Z$ are bounded and none of its forms is the trivial form, we see that the maximal $k$ such that $\zeta_{i}$ is the trivial form modulo $p^{k}$ is bounded. Remark C.1 in Appendix C, where we collect a number of elementary justifications in order not to break the flow of the exposition here, implies a bound of the form

$$
\alpha_{Z}\left(p^{k_{1}}, \ldots, p^{k_{s}}\right) \ll p^{-\max _{j} k_{j}},
$$


and thus

$$
\delta_{p} \ll \sum_{\substack{0 \leq k_{t+1}, \ldots, k_{t+s} \leq m \\ M:=\max k_{i} \geq m_{0}}} p^{-M}
$$

Bounding the number of tuples $\left(k_{1}, \ldots, k_{s}\right)$ satisfying $\max k_{i}=j$ crudely by $(j+1)^{s}$, we conclude that

$$
\begin{aligned}
\delta_{p} & \ll \sum_{j \geq m_{0}} p^{-j} j^{s} \\
& \ll \sum_{j \geq m_{0}} p^{-j / 2} \\
& \ll p p^{-m_{0} / 2} .
\end{aligned}
$$

Finally, this means that for $m \geq m_{0}$, we have

$$
\beta_{p}(m)=\mathbb{E}_{a \in\left(\mathbb{Z} / p^{m_{0}} \mathbb{Z}\right)^{d}} P(a) 1_{A_{1}\left(m_{0}, m_{0}\right)}+O\left(m_{0}^{s} p^{-m_{0} / 2}\right) .
$$

The same holds for $\beta_{p}(n)$, hence

$$
\beta_{p}(m)-\beta_{p}(n)=O\left(m_{0}^{s} p^{-m_{0} / 2}\right)
$$

and the conclusion follows.

Lemma A.2. Let $p$ be a prime. Then

$$
\mathbb{E}_{a \in\left(\mathbb{Z} / p^{\iota(p)} \mathbb{Z}\right)^{d}} \prod_{i=1}^{t} \Lambda_{p}\left(\psi_{i}(a)\right) \prod_{j=t+1}^{t+s} \frac{\rho_{f_{j}, \psi_{j}(a)}\left(p^{\iota(p)}\right)}{p^{\iota(p)}} 1_{\psi_{j}(a) \neq 0 \bmod p^{\iota(p)}}=\beta_{p}+O\left(\log ^{-C_{1} / 3} N\right)
$$

where $C_{1}$ is the constant appearing in (7), the definition of $\iota(p)$

Proof. We simply apply the proof of the above proposition with $m_{0}=\max \left(\iota(p), M_{0}\right)$. We use $m_{0}^{s} p^{-m_{0}} \ll p^{-m_{0} / 3}$, where the implied constant is independent of $m_{0}$ and $p_{0}$. This yields the desired result.

We now analyse the behaviour of $\beta_{p}$ as $p$ tends to infinity.

Lemma A.3. For primes $p$ tending to infinity,

$$
\beta_{p}=1+O\left(p^{-2}\right)
$$

Thus the product of the $\beta_{p}$ is convergent and

$$
\prod_{p \leq w(N)} \beta_{p}=\left(1+O\left(\frac{1}{w(N)}\right)\right) \prod_{p} \beta_{p}
$$

Proof. Assume $p$ is large enough so that $p$ does not divide the product $D_{t+1} \cdots D_{t+s}$ of the (negative) discriminants of our quadratic forms. 
Recall the notation $P(a)=P_{m}(a)$ from (24) and the sets $A_{1}=A_{1}(m, m)$ and $A_{2}=$ $A_{2}(m, m)$ introduced during the proof of Proposition A.1. As $m$ tends to $\infty$, we have

$$
\begin{aligned}
\beta_{p}+o(1) & =\mathbb{E}_{a \in\left(\mathbb{Z} / p^{m} \mathbb{Z}\right)^{d}} P(a) \\
& =\mathbb{E}_{a \in\left(\mathbb{Z} / p^{m} \mathbb{Z}\right)^{d}} P(a) 1_{A_{1}}(a)+\mathbb{E}_{a \in\left(\mathbb{Z} / p^{m} \mathbb{Z}\right)^{d}} P(a) 1_{A_{2}}(a) \\
& =\frac{1}{p^{m} d} \sum_{a \in A_{1}} P(a)+2^{t} O\left(s m^{s} p^{-m}\right) .
\end{aligned}
$$

To get this error term, we used Corollary C.4 and the triangle inequality to bound $\left|A_{2}\right|$, and the fact that $\rho_{f_{j}, \beta}\left(p^{m}\right) / p^{m} \ll m[16$, Lemma 6.3(c)] to bound $P(a)$. This error term tends to 0 as $m$ tends to infinity, and thus merges with the $o(1)$ of the left-hand side. Let us now consider the main term. Thanks to the choice of $p$ and the fact that the forms do not vanish at $a \bmod p^{m}$, we can use Lemma 6.3 from [16] which states that if $f$ is a PDBQF of discriminant $D$, and if $p$ is a prime which does not divide $D$, and if $\beta \neq 0 \bmod p^{m}$, then

$$
\frac{\rho_{f, \beta}\left(p^{m}\right)}{p^{m}}=\left(1-\chi_{D}(p) p^{-1}\right) \sum_{k=0}^{m} 1_{p^{k} \mid m} \chi_{D}\left(p^{k}\right) .
$$

Here $\chi_{D}$ is a real character modulo $p$, namely the Kronecker symbol [16, Lemma 2.1]. Thus

$$
\left.\beta_{p}=\lim _{m \rightarrow \infty} \mathbb{E}_{a \in\left(\mathbb{Z} / p^{m}\right.} \mathbb{Z}\right)^{d} \prod_{i=1}^{t} \Lambda_{p}\left(\psi_{i}(a)\right) \prod_{j=t+1}^{t+s}\left(1-\chi_{D_{j}}(p) p^{-1}\right) \sum_{k=0}^{m} 1_{p^{k} \mid \psi_{j}(a)} \chi_{D_{j}}\left(p^{k}\right)
$$

where we have obviously reintegrated the once excluded $a \in A_{2}$, because their sparsity ensures that they do not affect the limit. For $a \in\left(\mathbb{Z} / p^{m} \mathbb{Z}\right)^{d}$, we then write $a=a^{\prime}+p b$ with $b \in\left(\mathbb{Z} / p^{m-1} \mathbb{Z}\right)^{d}$ and $a^{\prime} \in[p]^{d}$. Thus the average $\mathbb{E}_{a}$ becomes

$$
\prod_{j=t+1}^{t+s}\left(1-\chi_{D_{j}}(p) p^{-1}\right) \mathbb{E}_{a \in[p]^{d}} \prod_{i=1}^{t} \Lambda_{p}\left(\psi_{i}(a)\right) \mathbb{E}_{b \in\left(\mathbb{Z} / p^{m-1} \mathbb{Z}\right)^{d}} \prod_{j=t+1}^{t+s} \sum_{k=0}^{m} 1_{p^{k} \mid \psi_{j}(a+p b)} \chi_{D_{j}}\left(p^{k}\right) .
$$

We expand the product of sums as follows

$$
\begin{aligned}
& \prod_{j=t+1}^{t+s} \sum_{k=0}^{m} 1_{p^{k} \mid \psi_{j}(a+p b)} \chi_{D_{j}}\left(p^{k}\right) \\
& =1+\sum_{j} \sum_{k_{j}=1}^{m} 1_{p^{k_{j}} \mid \psi_{j}(a+p b)} \chi_{D_{j}}\left(p^{k_{j}}\right)+\sum_{\substack{0 \leq k_{t+1}, \ldots, k_{t+s} \leq m \\
\text { at least two } k_{i}>0}} \prod_{p^{k_{j} \mid \psi_{j}(a+p b)} \chi_{D_{j}}\left(p^{k_{j}}\right)}
\end{aligned}
$$

according to whether we take no, one or several nonzero $k$. The expectation over $a$ from (28) then splits into three terms. The first one is

$$
\mathbb{E}_{a \in(\mathbb{Z} / p \mathbb{Z})^{d}} \prod_{i=1}^{t} \Lambda_{p}\left(\psi_{i}(a)\right)
$$


and he second one is

$$
\sum_{j=t+1}^{t+s} \sum_{k_{j}=1}^{m} \chi_{D_{j}}\left(p^{k_{j}}\right) \mathbb{E}_{a \in[p]^{d}} \prod_{i=1}^{t} \Lambda_{p}\left(\psi_{i}(a)\right) \mathbb{E}_{b \in\left(\mathbb{Z} / p^{m-1} \mathbb{Z}\right)^{d}} 1_{p^{k_{j}} \mid \psi_{j}(a+p b)} .
$$

Now we decompose $\psi_{j}(a+p b)=\psi_{j}(a)+p \dot{\psi}_{j}(b)$, where $\dot{\psi}$ is the linear part of $\psi$. If $p^{k_{j}}$ is to divide $\psi_{j}(a)+p \dot{\psi}_{j}(b)$, we need $p \mid \psi_{j}(a)$. Thus we can write, for each such $a$ fixed, $\psi_{j}(a+p b)=p \tilde{\psi}_{j}(b)$, where $\tilde{\psi}_{j}$ is again an affine-linear form whose linear part is $\dot{\psi}_{j}$. We then need $p^{k_{j}-1} \mid \tilde{\psi}_{j}(b)$. Because of Corollary C.5,

$$
\mathbb{E}_{b \in\left(\mathbb{Z} / p^{m-1} \mathbb{Z}\right)^{d}} 1_{p^{k_{j}-1} \mid \tilde{\psi}_{j}(b)}=p^{-k_{j}+1}
$$

so the expression (29) equals

$$
\sum_{j=t+1}^{t+s} \sum_{k_{j}=1}^{m} \chi_{D_{j}}\left(p^{k_{j}}\right) p^{-k_{j}} \mathbb{E}_{a \in[p]^{d}} \prod_{i=1}^{t} \Lambda_{p}\left(\psi_{i}(a)\right) p 1_{p \mid \psi_{j}(a)}
$$

To deal with the last term, which is

$$
\mathbb{E}_{a \in\left(\mathbb{Z} / p^{m} \mathbb{Z}\right)^{d}} \prod_{i=1}^{t} \Lambda_{p}\left(\psi_{i}(a)\right) \sum_{\substack{0 \leq k_{t+1}, \ldots, k_{t+s} \leq m \\ \text { at least two } k_{i}>0}} \prod_{j=t+1}^{t+s} 1_{p^{k_{j}} \mid \psi_{j}(a)} \chi_{D_{j}}\left(p^{k_{j}}\right)
$$

we crudely bound $\Lambda_{p}$ by 2 and $\chi_{D_{j}}$ by 1 . Recall the notation $Z$ from (26). Thus as $m$ tends to infinity, the expression (30) is bounded above by a constant times

$$
O\left(\sum_{\substack{k_{1}, \ldots, k_{s} \\ \text { at least two } k_{i}>0}} \alpha_{Z}\left(p^{k_{1}}, \ldots, p^{k_{s}}\right)\right)
$$

To bound this expression, we remember that $Z$ is a system of finite complexity. This implies, thanks to Proposition C.5, that for $p$ large enough 5 depending on $s, d, L$, we have

$$
\alpha_{Z}\left(p^{k_{1}}, \ldots, p^{k_{s}}\right) \leq p^{-\max _{i \neq j}\left(k_{i}+k_{j}\right)} \leq p^{-1-\max \left(k_{i}\right)}
$$

whenever at least two $k_{i}$ are nonzero. For any $k \geq 1$, there are at most $s(k+1)^{s-1} s$-tuples that satisfy $\max k_{i}=k$. Thus

$$
\sum_{\substack{k_{1}, \ldots, k_{s} \\ \text { at least two } k_{i}>0}} \alpha_{Z}\left(p^{k_{1}}, \ldots, p^{k_{s}}\right)=O\left(\sum_{k \geq 1} s k^{s-1} p^{-k-1}\right)=O_{s}\left(p^{-2}\right) .
$$

\footnotetext{
${ }^{5}$ We need $p$ to be large because for some small $p$, there could be two forms that, though affinely independent, become dependent when reduced modulo $p$. Such primes are called exceptional. The same need for large $p$ will appear again later.
} 
Putting these three terms together and letting $m$ tend to infinity, we get

$$
\begin{aligned}
\beta_{p}=\prod_{j=t+1}^{t+s}\left(1-\chi_{D_{j}}(p) p^{-1}\right)\left(\mathbb{E}_{a \in[p]^{d}} \prod_{i=1}^{t} \Lambda_{p}\left(\psi_{i}(a)\right)\right. \\
\left.\quad+\sum_{j=t+1}^{t+s} \mathbb{E}_{a \in[p]^{d}} p 1_{\psi_{j}(a)=0} \prod_{i=1}^{t} \Lambda_{p}\left(\psi_{i}(a)\right) \sum_{k=1}^{+\infty} \chi_{D_{j}}\left(p^{k}\right) p^{-k}\right)+O_{s, t}\left(p^{-2}\right)
\end{aligned}
$$

Green and Tao [8, Lemma 1.3] proved that $\mathbb{E}_{a \in[p]^{d}} \prod_{i=1}^{t} \Lambda_{p}\left(\psi_{i}(a)\right)=1+O_{t}\left(p^{-2}\right)$. Similarly, for any $j \in \llbracket t+1 ; t+s \rrbracket$, we have

$$
\begin{aligned}
\mathbb{E}_{a \in[p]^{d}} p 1_{p \mid \psi_{j}(a)} \prod_{i=1}^{t} \Lambda_{p}\left(\psi_{i}(a)\right) & \left.=p\left(\frac{p}{p-1}\right)^{t} \mathbb{P}\left(\left(\prod_{i=1}^{t} \psi_{i}(a), p\right)=1\right) \text { and } p \mid \psi_{j}(a)\right) \\
& =1+O\left(p^{-2}\right)
\end{aligned}
$$

because the probability is $p^{-1}\left(1-t / p+O\left(p^{-2}\right)\right)$ by linear independence. Moreover,

$$
\prod_{j=t+1}^{t+s}\left(1-\chi_{D_{j}}(p) p^{-1}\right)\left(1+\sum_{j=t+1}^{t+s} \sum_{k_{j}>0} \chi_{D_{j}}\left(p^{k_{j}}\right) p^{-k_{j}}\right)=1+O_{s}\left(p^{-2}\right)
$$

so that finally, plugging these estimates in (31), we obtain

$$
\beta_{p}=\prod_{j=t+1}^{t+s}\left(1-\chi_{D_{j}}(p) p^{-1}\right)\left(1+\sum_{j=t+1}^{t+s} \sum_{k_{j}>0} \chi_{D_{j}}\left(p^{k_{j}}\right) p^{-k_{j}}+O_{s, t}\left(p^{-2}\right)\right)=1+O\left(p^{-2}\right) .
$$

Here the implied constant depends on $t, d, s, L$ and the discriminants only. This last equation is exactly the claimed result.

\section{Appendix B. Verification of THE Linear FORMS CONDition}

This section is dedicated to the lengthy and technical proof of Proposition 3.7, that is, the verification that our majorant, introduced in Subsection 3.2.3, satisfies the linear forms condition. We loosely follow Matthiesen's proof in [16], taking inspiration of the more recent paper [2]. However, there is some flaw there, as the author overlooked the possibility that $u$ and $d m^{2} \epsilon$ may not be coprime; we provide, based on the earlier paper [15], a corrected version of these computations.

Compared to Matthiesens's articles, the introduction of the majorant for the von Mangoldt function adds factors of $\log R$ which will be cancelled during the Fourier transformation step. It also adds factors of $\frac{\phi(W)}{W}$ which remain untouched throughout the proof. And in the core of the calculation, it adds to the variables $d, m, e, u$ another variable $\ell$ also ranging among the integers whose prime factors are all greater than $w(N)$, which shall interact nicely with the other ones. The aim of the game is to dissociate the factors, that is, to transform the average of the product into the product of averages. 
Notational conventions for the proof. In order to somewhat lighten the formidable notation, we will not always specify the range on sums, products or integrals. In principle, the name of the variable alone should tell the reader what its range is. We list a few important conventions.

- The integer vector $n$ will always range in $\mathbb{Z}^{d} \cap K$.

- We put $\phi_{j}(n)=\bar{W} \psi_{j}(n)+b_{j}$, for $j \in[t+s]$, where $b_{j}$ lies in the set $B_{t, s}$ defined in Definition 2.2.

- For $i=1, \ldots, t$ and $k=1,2, \ell_{i, k}$ is a positive integer. Because it will always be a divisor of $\phi_{i}(n)$ which satisfies $\phi_{i}(n) \equiv b_{i} \bmod W$ and $\left(b_{i}, W\right)=1$ by definition of $B$, the prime factors of $\ell_{i, k}$ are all greater than $w(N)$.

- For $j=t+1, \ldots, t+s$ and $k=1,2, e_{j, k}$ is a positive integer in $\left\langle\mathcal{Q}_{k}\right\rangle$, where $\mathcal{Q}_{k}=\mathcal{Q}_{D_{k}}$. All its prime factors are greater than $w(N)$.

- For $j=t+1, \ldots, t+s, s_{j}$ will range from $2 / \gamma$ to $(\log \log N)^{3}$ and $i_{j}$ from $\log _{2} s-2$ to $6 \log \log \log N$, while $u_{j}$ ranges in $U\left(s_{j}, i_{j}\right)$. The $s_{j}$ should not be confused with $s$, the number of factors of the form $\nu_{\mathrm{Matt}, b}$. Notice that $i$ is also the standard name of the index ranging in $[t]$ but this should not cause any ambiguity.

- Occasionally we may want to write $e_{j}$ for $e_{j, 1}$ and $e_{j}^{\prime}=e_{j, 2}$; similarly $\ell_{i}=\ell_{i, 1}$ and $\ell_{i}^{\prime}=\ell_{i, 2}$. Moreover $\epsilon_{j}$ will be the least common multiple (lcm) of $e_{j}$ and $e_{j}^{\prime}$, while $\lambda_{i}$ will be the lcm of $\ell_{i}$ and $\ell_{i}^{\prime}$.

- For $j=t+1, \ldots, t+s$, the integer $d_{j}$ only has prime factors greater than $w(N)$ and lying in $\mathcal{P}_{j}$ where $\mathcal{P}_{j}=\mathcal{P}_{D_{j}}$.

- For $j=t+1, \ldots, t+s$, the integer $m_{j}$ only has prime factors greater than $w(N)$ and lying in $\mathcal{Q}_{j}$.

- A bold character denotes a vector; thus $\mathbf{e}=\left(e_{j, k}\right)_{\substack{j \in \llbracket t+1 ; t+s \rrbracket \\ k=1,2}}$ and again the range of such indices $i, k$ will frequently be omitted. Unfortunately, the symbol $\ell$ cannot be boldfaced, but this should not cause any ambiguity.

With these conventions, recalling the definitions (17) of $\Lambda_{\chi, R}$ and (20) of $r_{D, \gamma}$, we expand the left-hand side of (23) as

$$
\begin{aligned}
& \Omega=H \mathbb{E}_{n \in \mathbb{Z}^{d} \cap K} \prod_{i \in[t]} \sum_{\ell_{i}, \ell_{i}^{\prime}} \mu\left(\ell_{i}\right) \mu\left(\ell_{i}^{\prime}\right) \chi\left(\frac{\log \ell_{i}}{\log R}\right) \chi\left(\frac{\log \ell_{i}^{\prime}}{\log R}\right) 1_{\lambda_{i} \mid \phi_{i}(n)} \\
& \prod_{j=t+1}^{t+s} \sum_{s_{j}, i_{j}, u_{j}} 2^{s_{j}} 1_{u_{j} \mid \phi_{j}(n)} \sum_{d_{j}, m_{j}, e_{j}, e_{j}^{\prime}} 1_{d_{j} m_{j}^{2} \epsilon_{j} \mid \phi_{j}(n)} \mu\left(e_{j}\right) \mu\left(e_{j}^{\prime}\right) \chi\left(\frac{\log e_{j}}{\log R}\right) \chi\left(\frac{\log e_{j}^{\prime}}{\log R}\right) \chi\left(\frac{\log d_{j}}{\log R}\right) \chi\left(\frac{\log m_{j}}{\log R}\right) .
\end{aligned}
$$

The initial factor $H$ is defined by

$$
H=\left(\log R \frac{\phi(W)}{W}\right)^{t} \prod_{j=t+1}^{t+s} C_{D_{j}, \gamma}^{-1}
$$


Proving Proposition 3.7 means proving that

$$
\Omega=1+O_{D}\left(\frac{N^{d-1+O_{D}(\gamma)}}{\operatorname{Vol}(K)}\right)+o_{D}(1) .
$$

Write $\Omega=H \Omega^{\prime}$. Notice that $H=\Omega / \Omega^{\prime}=O\left((\log R)^{t}\right)=O\left((\log N)^{t}\right)$. We now work on $\Omega^{\prime}$. It is an average over $n$ of $t+s$ products, and we aim at transforming it into a product of $t+s$ averages. We will remember to multiply the error terms obtained during the transformation of this average by $(\log N)^{t}$ to obtain error terms for $S$.

We observe that when $u_{j}, d_{j}, m_{j}, e_{j}, e_{j}^{\prime}$ divide $\phi_{j}(n)$ and $u_{j} \operatorname{satisfies} \operatorname{gcd}\left(u_{j}, \phi_{j}(n) / u_{j}\right)=1$, there exists, for $x$ equal to any of the symbols $e, e^{\prime}, d, m$, a unique decomposition

$$
x_{j}=\tilde{x}_{j} v_{j, x} \text { with } \operatorname{gcd}\left(\tilde{x}_{j}, u_{j}\right)=1 \text { and } v_{j, x} \mid u_{j} .
$$

We would very much like to perform this decomposition, but not every term satisfies the required coprimality condition. However, the following claim shows that we can pretend it does at a small cost.

Claim 1. The summands in (32) satisfying $\operatorname{gcd}\left(u_{j}, \phi_{j}(n) / u_{j}\right)>1$ for some $j$ or $\operatorname{gcd}\left(u_{j}, u_{i}\right)>1$ for some $i \neq j$ contribute only $O\left(N^{-(\log \log N)^{-3} / 8}\right)$.

Proof. We write the contribution of these summands as

$$
S=H \sum_{\mathbf{i}, \mathbf{s}} \prod_{j=t+1}^{t+s} 2^{s_{j}} \mathbb{E}_{n} a_{n}
$$

where

$$
\begin{aligned}
a_{n}=a_{n, \mathbf{i}, \mathbf{s}}=\sum_{\mathbf{u}} 1_{\begin{array}{c}
\exists j \mid \operatorname{gcd}\left(u_{j}, \phi_{j}(n) / u_{j}\right)>1 \\
\text { or } \exists i \neq j \mid \operatorname{gcd}\left(u_{j}, u_{i}\right)>1
\end{array}} \sum_{\mathbf{d}, \mathbf{m}, \mathbf{e}, \ell} \prod_{i=1}^{t} \mu\left(\ell_{i}\right) \mu\left(\ell_{i}^{\prime}\right) \chi\left(\frac{\log \ell_{i}}{\log R}\right) \chi\left(\frac{\log \ell_{i}^{\prime}}{\log R}\right) 1_{\lambda_{i} \mid \phi_{i}(n)} \\
\prod_{j=t+1}^{t+s} \mu\left(d_{j}\right) \mu\left(\ell_{j}\right) \mu\left(\ell_{j}^{\prime}\right) \chi\left(\frac{\log e_{i}}{\log R}\right) \chi\left(\frac{\log e_{i}^{\prime}}{\log R}\right) 1_{\Delta_{j} \mid \phi_{j}(n)}
\end{aligned}
$$

with the notation $\Delta_{j}=\operatorname{gcd}\left(u_{j}, d_{j} m_{j}^{2} e_{j}\right)$. To bound $\mathbb{E}_{n} a_{n}$, we apply the simple rule, based on Cauchy-Schwarz, that

$$
\left(\mathbb{E}_{n \in \mathbb{Z}^{d} \cap K} a_{n}\right)^{2} \leq \mathbb{P}_{n}\left(a_{n} \neq 0\right) \mathbb{E}_{n} a_{n}^{2}
$$

Now if $a_{n} \neq 0$ then either the value of one of the $s$ last linear forms $\phi_{i}(n)$ has a repeated prime factor, or the values of two of the $s$ last linear forms have a common prime factor. Such a prime $p$ is a factor of some $u_{i}$, which, by Definition 3.3 , only has prime factors larger than $N^{1 /(\log \log N)^{3}}$ and satisfies $u_{i} \leq N^{\gamma}$ (see [15, Proposition 4.2]). Thus $p$ certainly lies between $N^{1 /(\log \log N)^{3}}$ and $N^{\gamma}$. Using the triangle inequality, we get

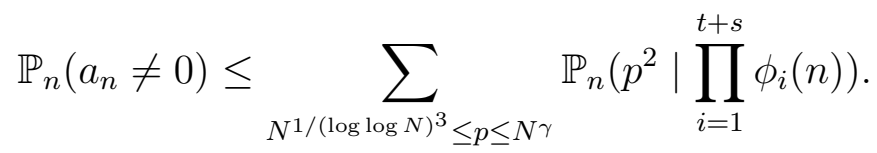

Moreover, the primes $p$ in this range are not exceptional primes, i.e. primes modulo which the linear forms are affinely dependent. Indeed, such primes, thanks to the $W$-trick 
and the fact that no two of the original linear forms $\psi_{i}$ are affinely dependent, are all $O(w(N))=O(\log \log N)$. Thus

$$
\mathbb{P}_{n}\left(p^{2} \mid \prod_{i} \phi_{i}(n)\right) \ll p^{-2}+O\left(\frac{N^{\prime d-1}}{\operatorname{Vol}(K)}\right)=p^{-2}+O\left(p^{2} \frac{N^{d-1}}{\operatorname{Vol}(K)}\right),
$$

according to Proposition C.6 and the fact that $\left|K \cap \mathbb{Z}^{d}\right| \sim \operatorname{Vol}(K) 6$ Hence

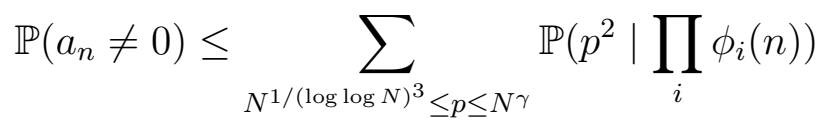

$$
\begin{aligned}
& \ll \sum_{p \geq N^{1 /(\log \log N)^{3}}} p^{-2}+\frac{N^{d-1}}{\operatorname{Vol}(K)} \sum_{p \leq N^{\gamma}} p^{2} \\
& \ll N^{-1 /(\log \log N)^{3}}+\frac{N^{3 \gamma+d-1}}{\operatorname{Vol}(K)} .
\end{aligned}
$$

Assuming that $\gamma$ is small enough (less than $1 / 3$ ), the second term is $O\left(N^{-c}\right)$ with $c>0$ so it is negligible with respect to the first one.

We then bound $\mathbb{E}_{n} a_{n}^{2}$ quite crudely as follows

$$
\begin{aligned}
\mathbb{E}_{n} a_{n}^{2} & \leq \mathbb{E}_{n}\left(\sum_{\mathbf{d}, \mathbf{m}, \mathbf{e}, \ell, \mathbf{u}} \prod_{i=1}^{t} 1_{\lambda_{i} \mid \phi_{i}(n)} \prod_{j=t+1}^{t+s} 1_{\Delta_{j} \mid \phi_{j}(n)}\right)^{2} \\
& \ll \prod_{i=1}^{t}\left(\mathbb{E}_{n}\left(\sum_{\ell_{i}, \ell_{i}^{\prime}} 1_{\lambda_{i} \mid \phi_{i}(n)}\right)^{2(t+s)}\right)^{1 /(t+s)} \prod_{j=t+1}^{t+s}\left(\mathbb{E}_{n}\left(\sum_{d_{j}, m_{j}, e_{j}, e_{j}^{\prime}, u_{j}} 1_{\Delta_{j} \mid \phi_{j}(n)}\right)^{2(t+s)}\right)^{1 /(t+s)} \\
& \ll(\log N)^{O_{t, s}(1)} .
\end{aligned}
$$

The first inequality is a consequence of the fact that $|\mu| \leq 1$ and $|\chi| \leq 1$. The second one is Hölder's. The last one follows from bounds of Matthiesen [15, Lemma 3.1] on moments of the divisor function, and the observation that for instance $\sum_{\ell_{i}, \ell_{i}^{\prime}} 1_{\lambda_{i} \mid \phi_{i}(n)} \leq \tau(\phi(n))^{2}$. Thus $\left|\mathbb{E}_{n} a_{n}\right| \ll N^{-(\log \log N)^{-3} / 4}$. Summing now over $\mathbf{i}, \mathbf{s}$ and multiplying by $H$, we get $|S| \leq N^{-(\log \log N)^{-3} / 8}$ as desired. This concludes the proof of Claim 1 .

Thus to evaluate (32), we shall pretend all summands satisfy the coprimality condition, transform them under this hypothesis, and then reintegrate the formerly excluded terms, which generates an error term of size $O\left(N^{-(\log \log N)^{-3} / 8}\right)$. So (32) is equal to

\footnotetext{
${ }^{6}$ Here, we assume that $\operatorname{Vol}(K) \gg N^{\prime d}$ or at least that $N^{\prime d-1}=o(\operatorname{Vol}(K))$. Indeed, in the statement of the main theorem, we could also add the assumption that $\operatorname{Vol}(K) \gg N^{d}$ because otherwise the error term is not smaller than the main term.
} 


$$
\begin{aligned}
\sum_{\mathbf{i}, \mathbf{s}} & \sum_{\mathbf{u}}^{\prime} \mathbb{E}_{n} \prod_{i \in[t], k=1,2} \sum_{\ell_{i, k}} \mu\left(\ell_{i, k}\right) \chi\left(\frac{\log \ell_{i, k}}{\log R}\right) 1_{\lambda_{i} \mid \phi_{i}(n)} \prod_{j=t+1}^{t+s} 2^{s_{j}} \sum_{d_{j}, e_{j}, e_{j}^{\prime}, m_{j} \text { coprime to } u_{j}} \\
& \prod_{\substack{v_{j, d}, v_{j, m}, v_{j, e}, v_{j, e^{\prime}} \\
\text { divisors of } u_{j} \in\left\{d_{j}, e_{j}, e_{j}^{\prime}, m_{j}\right\}}} \chi\left(\frac{\log x_{j} v_{j, x}}{\log R}\right) \mu\left(e_{j} v_{j, e}\right) \mu\left(e_{j} v_{j, e^{\prime}}\right) 1_{u_{j} d_{j} \epsilon_{j} m_{j}^{2} \mid \phi_{j}(n)}+O\left(N^{\left.-(\log \log N)^{-3 / 8}\right)}\right.
\end{aligned}
$$

where the dashed sum indicates a sum over vectors whose entries are coprime.

Above and from now on, the vectors $\mathbf{d}, \mathbf{e}, \mathbf{m}$ are assumed to be entrywise coprime to the vector $\mathbf{u}$. Thus we can perform the decomposition (33). The vector $\mathbf{v}$ stands for $\left(v_{j, x}\right)_{x \in\left\{d, e, e^{\prime}, m\right\}, j \in \llbracket t+1 ; t+s \rrbracket}$ where we impose for every $j$ the conditions $v_{j, x} \mid u_{j}$ and $v_{j, d} \in$ $\left\langle\mathcal{P}_{j}\right\rangle, v_{j, m} \in\left\langle\mathcal{Q}_{j}\right\rangle, v_{j, e} \in\left\langle\mathcal{P}_{j}\right\rangle$. Furthermore, we shall use the notation

$$
q_{j}=\left\{\begin{array}{cc}
\lambda_{j} & \text { if } j \in[t] \\
u_{j} d_{j} \epsilon_{j} m_{j}^{2} & \text { if } j \in \llbracket t+1 ; t+s \rrbracket .
\end{array}\right.
$$

Claim 2. The main term of (34) is equal to

$$
\begin{aligned}
& \sum_{\mathbf{i}, \mathbf{s}} \sum_{\mathbf{u}} \sum_{\mathbf{d}, \mathbf{e}, \mathbf{m}, \ell} \alpha\left(q_{1}, \ldots, q_{t+s}\right) \\
& \sum_{\mathbf{v}} \prod_{i \in[t], k=1,2} \mu\left(\ell_{i, k}\right) \chi\left(\frac{\log \ell_{i, k}}{\log R}\right) \prod_{j \in \llbracket t+1 ; t+s \rrbracket} \frac{2^{s_{j}}}{u_{j}} \mu\left(e_{j} v_{j, e}\right) \mu\left(e_{j} v_{j, e^{\prime}}\right) \prod_{x_{j} \in\left\{d_{j}, e_{j}, e_{j}^{\prime}, m_{j}\right\}} \chi\left(\frac{\log x_{j} v_{j, x}}{\log R}\right)
\end{aligned}
$$

up to an error of size $O\left(N^{d-1+O(\gamma)} / \operatorname{Vol}(K)\right)$.

We remark that this error term, after multiplication by the initial factor $H=O\left((\log N)^{t}\right)$, is still of the same magnitude.

Proof. First, we apply Lemma C.2

$$
\mathbb{E}_{n \in \mathbb{Z}^{d} \cap K} \prod_{i=1}^{t} 1_{\lambda_{i} \mid \phi_{i}(n)} \prod_{j=t+1}^{t+s} 1_{u_{j} d_{j} m_{j}^{2} \epsilon_{j} \mid \phi_{j}(n)}=\alpha\left(q_{1}, \ldots, q_{t+s}\right)+O\left(N^{d-1+O(\gamma)} / \operatorname{Vol}(K)\right) .
$$

To explain the error term, observe that for any set of tuples bringing a nonzero contribution, for any $j \in[t+s]$, we have $q_{j}=N^{O(\gamma)}$ because $d_{j}, m_{j}, e_{j}, e_{j}^{\prime} \leq N^{\gamma}$ and $u_{j} \leq N^{\gamma}$. To bound the contribution of this error term to the sum defining the main term of (34), we simply notice that the number of terms is $O\left(N^{\gamma}\right)$ anyway, that the $\mu$ and $\chi$ factors are 1-bounded, and that $2^{s_{j}}$ is always $o\left(N^{\gamma}\right)$ because $s \leq(\log \log N)^{3}$.

Notice that we can also exclude summands for which $\operatorname{gcd}\left(\lambda_{i}, u_{j}\right)>1$ for some $i \in[t]$ and $j \in \llbracket t+1 ; t+s \rrbracket$ because of the very same argument as in Claim 1. For summands satisfying to the contrary $\operatorname{gcd}\left(\lambda_{i}, u_{j}\right)=1$, by multiplicativity of $\alpha$ and because of the other 
implicit coprimality conditions, we can write

$$
\alpha\left(q_{1}, \ldots, q_{t+s}\right)=\frac{\alpha\left(\left(\lambda_{i}\right)_{i \in[t]},\left(d_{j} m_{j}^{2} \epsilon_{j}\right)_{j \in \llbracket t+1 ; t+s \rrbracket}\right)}{\prod_{j} u_{j}} .
$$

This concludes the proof of this claim with a dashed sum on $u$ instead of the normal sum, and a sum on $\ell$ restricted to tuples satisfying $\operatorname{gcd}\left(\lambda_{i}, u_{j}\right)>1$ for all $i$ and $j$. We can reintegrate now the formerly excluded terms because they have a negligible contribution anyway, so Claim 2 is proven.

Having removed the terms $u_{j}$ from the local density $\alpha$, we reset the definition of $q_{j}$ as follows

$$
q_{j}=\left\{\begin{array}{cc}
\lambda_{j} & \text { if } j \in[t] \\
d_{j} \epsilon_{j} m_{j}^{2} & \text { if } j \in \llbracket t+1 ; t+s \rrbracket .
\end{array}\right.
$$

From now on, we fix vectors $\mathbf{i}, \mathbf{s}$ in their usual ranges, and consider the individual terms

$$
\begin{aligned}
& \sum_{\mathbf{u}} \sum_{\mathbf{d}, \mathbf{e}, \mathbf{m}, \ell} \alpha\left(q_{1}, \ldots, q_{t+s}\right) \prod_{i \in[t], k=1,2} \mu\left(\ell_{i, k}\right) \chi\left(\frac{\log \ell_{i, k}}{\log R}\right) \\
& \sum_{\mathbf{v}} \prod_{j \in \llbracket t+1 ; t+s \rrbracket} \frac{2^{s_{j}}}{u_{j}} \mu\left(e_{j} v_{j, e}\right) \mu\left(e_{j}^{\prime} v_{j, e^{\prime}}\right) \prod_{x_{j} \in\left\{d_{j}, e_{j}, e_{j}^{\prime}, m_{j}\right\}} \chi\left(\frac{\log x_{j} v_{j, x}}{\log R}\right)
\end{aligned}
$$

We now use the Fourier transform. Letting $\theta$ be the Fourier transform of the smooth compactly supported function $x \mapsto e^{x} \chi(x)$, it is well known that

$$
\forall A>0 \quad \theta(\xi) \ll_{A}(1+|\xi|)^{-A} .
$$

This allows us to reconstruct $\chi$ from $\theta$ as an integral over the compact interva 7

$$
I=\left\{\xi \in \mathbb{R}|| \xi \mid \leq \log ^{1 / 2} R\right\}
$$

at the cost of a tolerable error; more precisely, for any $A>0$, we have

$$
\begin{aligned}
\chi\left(\frac{\log x}{\log R}\right) & =\int_{\mathbb{R}} x^{-\frac{1+i \xi}{\log R}} \theta(\xi) d \xi \\
& =\int_{I} x^{-\frac{1+i \xi}{\log R}} \theta(\xi) d \xi+O\left(x^{-\frac{1}{\log R}} \log ^{-A} R\right) .
\end{aligned}
$$

When plugging this into our sum, we need $4 s+2 t$ real variables $\xi_{j, k}$ with $k=1, \ldots, 4$ for $j=t+1, \ldots, t+s$ and $k=1,2$ for $j=1, \ldots, t$. Collectively, they form the vector $\boldsymbol{\Xi}$. Furthermore, we write $z_{j, k}=\left(1+i \xi_{j, k}\right) /(\log R)$. We sometimes allow, for a function $f$, the slight abuse of notation

$$
\prod_{j, k} f\left(\xi_{j, k}\right)=\prod_{i \in[t], k \in[2]} f\left(\xi_{i, k}\right) \prod_{j \in[t+s] \backslash[t], k \in[4]} f\left(\xi_{j, k}\right),
$$

\footnotetext{
${ }^{7}$ We prefer integrating over a compact set, in order to be able to easily swap summation and integration using Fubini's theorem.
} 
and write

$$
\theta(\boldsymbol{\Xi})=\prod_{j, k} \theta\left(\xi_{j, k}\right)
$$

We introduce the notation $\tilde{x}_{j}=x_{j} v_{j, x}$ for $x$ equal to any of the symbols $e, e^{\prime}, d, m$, and $\mathbf{v}_{\mathbf{i}}=\left(v_{i, d}, v_{i, e}, v_{i, e^{\prime}}, v_{i, m}\right)$. For any fixed values of the tuples $\mathbf{s}, \mathbf{i}, \mathbf{u}, \mathbf{v}, \mathbf{d}, \mathbf{m}, \mathbf{e}, \ell$ we write

$$
M=\prod_{i \in[t], k=1,2} \mu\left(\ell_{i, k}\right) \prod_{j \in \llbracket t+1 ; t+s \rrbracket} \frac{2^{s_{j}}}{u_{j}} \mu\left(e_{j} v_{j, e}\right) \mu\left(e_{j}^{\prime} v_{j, e^{\prime}}\right) .
$$

Finally, we introduce

$$
F_{\mathbf{d}, \mathbf{m}, \mathbf{e}, \ell}(\boldsymbol{\Xi})=F(\boldsymbol{\Xi})=\theta(\boldsymbol{\Xi}) \prod_{j>t} \tilde{e}_{j, 1}^{-z_{j, 1}} \tilde{e}_{j, 2}^{-z_{j, 2}} \tilde{d}_{j}^{-z_{j, 3}} \tilde{m}_{j}^{-z_{j, 4}} \prod_{i \in[t]} \ell_{i, 1}^{-z_{i, 1}} \ell_{i, 2}^{-z_{i, 2}}
$$

We now insert (38) into the expression (36) to get

$$
\sum_{\mathbf{d}, \mathbf{m}, \mathbf{e}, \ell} \alpha\left(q_{1}, \ldots, q_{t+s}\right) \sum_{\mathbf{u}, \mathbf{v}} M\left(\int_{I^{4 s+2 t}} F(\boldsymbol{\Xi}) d \boldsymbol{\Xi}+O\left((\log R)^{-A}\left(\prod_{i, j, k} \tilde{e}_{j, k} \ell_{i, k} \tilde{d}_{j} \tilde{m}_{j}\right)^{-1 / \log R}\right)\right) .
$$

Here the term arising from the big oh will not matter too much, thanks to the following claim.

Claim 3. For $A>0$ large enough,

$$
H \sum_{\mathbf{s}, \mathbf{i}} \sum_{\mathbf{u}, \mathbf{v}} \sum_{\mathbf{d}, \mathbf{m}, \mathbf{e}, \ell} \alpha\left(q_{1}, \ldots, q_{t+s}\right) M \log ^{-A} R\left(\prod_{i \in[t], k=1,2} \ell_{i, k} \prod_{j>t} \tilde{e}_{j, k} \tilde{m}_{j} \tilde{d}_{j}\right)^{-1 / \log R}=o(1) .
$$

Proof. Matthiesen [15, Proposition 4.2] showed that

$$
\sum_{\mathbf{s}, \mathbf{i}} \prod_{j=t+1}^{t+s} \sum_{u_{j} \in U\left(s_{j}, i_{j}\right)} \frac{2^{s_{j}}}{u_{j}}=O(1)
$$

On the other hand, we can suppress the sum over $\mathbf{v}$ by reintegrating into the sum over $\mathbf{d}, \mathbf{m}, \mathbf{e}$ the summands not termwise coprime to $\mathbf{u}$. We can then drop the $\sim$ on the variables. We put $q_{j}^{\prime}=\ell_{j} \ell_{j}^{\prime}$ for $j \in[t]$ and $q_{j}=e_{j} e_{j}^{\prime} d_{j} m_{j}$ for $j \in[t+s] \backslash[t]$. By multiplicativity,

$$
\begin{aligned}
\sum_{\mathbf{d}, \mathbf{m}, \mathbf{e}, \ell} \alpha\left(q_{1}, \ldots, q_{t+s}\right)\left(\prod_{\substack{i \in[t] \\
k=1,2}} \ell_{i, k} \prod_{j>t} e_{j, k} m_{j} d_{j}\right)^{-\frac{1}{\log R}} & =\sum_{\mathbf{d}, \mathbf{m}, \mathbf{e}, \ell p^{a_{i}} \| q_{i}} \alpha\left(p^{a_{1}}, \ldots, p^{a_{t+s}}\right) \prod_{\substack{j \in[t+s] \\
p_{j}^{\prime}} q_{j}^{\prime}} p^{-\frac{a_{j}^{\prime}}{\log R}} \\
& \leq \sum_{\mathbf{d}, \mathbf{m}, \mathbf{e}, \ell p^{a_{i}} \| q_{i}} p^{-\max a_{i}\left(1+2 \log ^{-1} R\right)} \\
& \leq \prod_{p}\left(1-p^{-\left(1+2 \log ^{-1} R\right)}\right)^{-O(t+s)} \\
& \ll \log ^{O(t+s)} N .
\end{aligned}
$$


Here we used $a_{j}^{\prime} \geq a_{j} / 2$, Corollary C.4 and a crude bound $k^{O(t+s)}$ for the number of tuples $a_{i}$ satisfying $\max _{i} a_{i}=k$. The last inequality follows from a well-known estimate for the Zeta function near 1, namely

$$
\zeta(x)=O\left(\frac{1}{x-1}\right)
$$

Given that $H=O\left(\log ^{t} N\right)$, the claim follows for $A$ large enough depending on $t$ and $s$ only.

We are left to deal with

$$
\sum_{\mathbf{d}, \mathbf{m}, \mathbf{e}, \ell} \alpha\left(q_{1}, \ldots, q_{t+s}\right) \sum_{\mathbf{u}, \mathbf{v}} M \int_{I^{4 s+2 t}} F(\boldsymbol{\Xi}) d \boldsymbol{\Xi} .
$$

We now swap the summation $\sum_{\mathbf{d}, \mathbf{m}, \mathbf{e}, \ell}$ and the integration over the compact set $I^{4 s+2 t}$, using Fubini's theorem. This causes no problem because the sum is absolutely convergent; we are not explicitly going to prove the absolute convergence, but it follows from the bounds we are going to derive in the proof of the next claim.

We also continue swapping summation and multiplication, by enforcing at little cost an extra coprimality condition: we show we can restrict to tuples where $\left(d_{j} m_{j} \epsilon_{j}, \lambda_{i}\right)=1$ for all $i, j$ and $\left(d_{i} m_{i} \epsilon_{i}, d_{j} m_{j} \epsilon_{j}\right)=1$ for all $i \neq j$. We need another, more subtle argument to impose this coprimality compared to the coprimality condition involving the variables $u_{j}$ in Claim 1, because a crucial ingredient of the proof of this claim was that the prime factors involved were all at least $N^{(\log \log N)^{-3}}$, an assumption we do not have for $d, m, e$.

Claim 4. Let $\mathbf{s}, \mathbf{i}, \mathbf{u}, \mathbf{v}$ be fixed vectors of integers satisfying the usual conditions. Then we have

$$
\begin{aligned}
& \sum_{\mathbf{d}, \mathbf{m}, \mathbf{e}, \ell} \alpha\left(q_{1}, \ldots, q_{t+s}\right) F(\boldsymbol{\Xi}) \prod_{i, j} \mu\left(\ell_{i, 1}\right) \mu\left(\ell_{i, 2}\right) \mu\left(\tilde{e}_{j, 1}\right) \mu\left(\tilde{e}_{j, 2}\right) \\
& =\left(1+O\left(w(N)^{-1 / 2}\right)\right) \sum_{\mathbf{d}, \mathbf{m}, \mathbf{e}, \ell}^{\prime} \alpha\left(q_{1}, \ldots, q_{t+s}\right) F(\boldsymbol{\Xi}) \prod_{i, j} \mu\left(\ell_{i, 1}\right) \mu\left(\ell_{i, 2}\right) \mu\left(\tilde{e}_{j, 1}\right) \mu\left(\tilde{e}_{j, 2}\right),
\end{aligned}
$$

where the dashed sum is retricted to tuples satisfying $\left(d_{j} m_{j} \epsilon_{j}, \lambda_{i}\right)=1$ for all $i, j$ and $\left(d_{i} m_{i} \epsilon_{i}, d_{j} m_{j} \epsilon_{j}\right)=1$ for all $i \neq j$.

Proof. The goal is to bound the contribution of the entries failing the coprimality conditions. To achieve this, we exploit the multiplicativity of each summand $T(\mathbf{d}, \mathbf{m}, \mathbf{e}, \ell)$, which is a term of the form

$$
\alpha\left(q_{1}, \ldots, q_{t+s}\right) \prod_{i=1}^{t} \ell_{i, 1}^{-z_{i, 1}} \ell_{i, 2}^{-z_{i, 2}} \mu\left(\ell_{i, 1}\right) \mu\left(\ell_{i, 2}\right) \prod_{j=t+1}^{t+s} \tilde{e}_{j, 1}^{-z_{j, 1}} \tilde{e}_{j, 2}^{-z_{j, 2}} \tilde{d}_{j}^{-z_{j, 3}} \tilde{m}_{j}^{-z_{j, 4}} \mu\left(\tilde{e}_{j, 1}\right) \mu\left(\tilde{e}_{j, 2}\right),
$$

in order to write it as a product over primes; only primes greater than $w(N)$ need be considered, as smaller ones have no chance of dividing any of the parameters. We can even 
partition the primes $p$ into two classes, according to whether $p$ divides a single $q_{j}$ or at least two of them. Thus, the summand $T$ can be written as

$$
\prod_{p \text { dividing a single } q_{j}} \alpha\left(\left(p^{v_{p}\left(q_{j}\right)}\right)_{j}\right) A_{p} \prod_{p \text { dividing at least two } q_{j}} \alpha\left(\left(p^{v_{p}\left(q_{j}\right)}\right)_{j}\right) B_{p}
$$

where $A_{p}$ and $B_{p}$ are complex numbers of modulus at most one and $v_{p}$ is the $p$-adic valuation. For any given tuples $\mathbf{d}, \mathbf{m}, \mathbf{e}, \ell$ and $j \in[s+t]$, we write $\kappa_{j}=\prod_{p \text { dividing at least two } q_{j}} p^{v_{p}\left(q_{j}\right)}$. Thus

$$
p\left|\kappa_{i} \Rightarrow p\right| \prod_{j \neq i} \kappa_{j} .
$$

We now arrange the summands $T(\mathbf{d}, \mathbf{m}, \mathbf{e}, \ell)$ according to their tuples $\left(\kappa_{1}, \ldots, \kappa_{t+s}\right)$. Let us fix such a tuple $\left(\kappa_{1}, \ldots, \kappa_{t+s}\right)$. The sum of summands $T$ corresponding to this tuple is

$$
\begin{aligned}
& \alpha\left(\kappa_{1}, \ldots, \kappa_{t+s}\right)\left|\sum_{\substack{\mathbf{d}, \mathbf{m}, \mathbf{e}, \ell \\
\forall j\left(q_{j}, \kappa_{j}\right)=1}}^{\prime} \alpha\left(q_{1}, \ldots, q_{t+s}\right) \prod_{\substack{j=t+1 \\
k=1,2}}^{t+s} \tilde{e}_{j, k}^{-z_{j, k}} \mu\left(\tilde{e}_{j, k}\right) \tilde{d}_{j}^{-z_{j, 3}} \tilde{m}_{j}^{-z_{j, 4}} \prod_{i \in[t]} \ell_{i, k}^{-z_{i, k}} \mu\left(\ell_{i, k}\right)\right| \\
& \leq \alpha\left(\left(\kappa_{j}\right)\right) \prod_{p \mid \prod_{j} \kappa_{j}}\left(1+O\left(p^{-1}\right)\right)\left|\sum_{\mathbf{d}, \mathbf{m}, \mathbf{e}, \ell}^{\prime} \alpha\left(\left(q_{j}\right)\right) \prod_{\substack{j=t+1 \\
k=1,2}}^{t+s} \tilde{e}_{j, k}^{-z_{j, k}} \mu\left(\tilde{e}_{j, k}\right) \tilde{d}_{j}^{-z_{j, 3}} \tilde{m}_{j}^{-z_{j, 4}} \prod_{i \in[t]} \ell_{i, k}^{-z_{i, k}} \mu\left(\ell_{i, k}\right)\right|
\end{aligned}
$$

by Möbius inversion (or inclusion-exclusion) and the triangle inequality. Next we evaluate the sum of all terms of the form $\alpha\left(\kappa_{1}, \ldots, \kappa_{t+s}\right) \prod_{p \mid \prod_{j} \kappa_{j}}\left(1+O\left(p^{-1}\right)\right)$. Using multiplicativity, we can write them as products over primes, all these primes being larger than $w(N)$. Thus, a crude bound is

$$
\prod_{p>w(N)}\left(1+\sum_{\begin{array}{c}
a_{1}, \ldots, a_{t+s} \\
\text { at least two } a_{i}>0
\end{array}} O\left(a_{1}^{2}+\cdots+a_{t}^{2}+a_{t+1}^{4}+\cdots+a_{t+s}^{4}\right) \alpha\left(\left(p^{a_{i}}\right)\right)\left(1+O\left(p^{-1}\right)\right)\right)-1
$$

where we have used the simple bound $\tau_{k}\left(p^{a_{i}}\right) \ll a_{i}^{k-1}$ for $k=3$ (because of $\lambda_{i}=\lambda_{i} / \ell_{i} \cdot \lambda_{i} / \ell_{i}^{\prime}$. $\ell_{i} \ell_{i}^{\prime} / \lambda_{i}$, hence the number of occurrences of $\lambda_{i}$ is bounded by the number of decompositions of it into three factors) and for $k=5$ (because of $d_{j} m_{j}^{2} \epsilon_{j}=d_{j} \cdot m_{j}^{2} \cdot \epsilon_{j} / e_{j} \cdot \epsilon_{j} / e_{j}^{\prime} \cdot e_{j} e_{j}^{\prime} / \epsilon_{j}$ ). The requirement that at least two $a_{i}$ be positive comes from the very definition of $\kappa_{i}$. Notice that the -1 is here to remove the 1 arising from $\alpha(1, \ldots, 1)$. To further bound this expression, we first bound $a_{i}^{2}$ by $a_{i}^{4}$ and recall that the number of tuples $\left(a_{1}, \ldots, a_{t+s}\right)$ satisfying $\max a_{i}=k$ is at most $t^{\prime}(k+1)^{t^{\prime}-1}$ (with $t^{\prime}=t+s$ ). For such tuples, we have $\sum_{i} a_{i}^{4} \leq t^{\prime} k^{4}$ and since the system is of finite complexity and at least two $a_{i}$ are nonzero, 
$\alpha\left(\left(p^{a_{i}}\right)_{i \in[t+s]}\right) \leq p^{-k-1}$ according to Proposition C.5. Thus

$$
\sum_{\substack{a_{1}, \ldots, a_{t+s} \\ \text { at least two } a_{i}>0}} O\left(a_{1}^{2}+\cdots+a_{t}^{2}+a_{t+1}^{4}+\cdots+a_{t+s}^{4}\right) \alpha\left(\left(p^{a_{i}}\right)\right)\left(1+O\left(p^{-1}\right)\right)
$$

is bounded by

$$
\sum_{k \geq 1} p^{-k-1} t^{\prime 2} k^{t^{\prime}+3} \ll \sum_{k \geq 1} p^{-3 k / 4-1} \ll p^{-3 / 2}
$$

the first inequality being provided by obvious growth comparisons valid for large $p$ (we may assume $N$ to be large enough for $p>w(N)$ to satisfy automatically this condition). Since

$$
\prod_{p>w(N)}\left(1+p^{-3 / 2}\right)-1 \leq \sum_{n>w(N)} n^{-3 / 2} \ll w(N)^{-1 / 2}
$$

Claim 4 follows.

The extra coprimality condition that Claim 4 allows us to assume enables us to write $\alpha$ as the product of the reciprocals of its arguments, resulting in

$$
\begin{aligned}
& \sum_{\mathbf{d}, \mathbf{m}, \mathbf{e}, \ell}^{\prime} \alpha\left(q_{1}, \ldots, q_{t+s}\right) \prod_{j=t+1}^{t+s} \frac{\mu\left(e_{j}\right) \mu\left(e_{j}^{\prime}\right)}{\epsilon_{j}} e_{j, 1}^{-z_{j, 1}} e_{j, 2}^{-z_{j, 2}} d_{j}^{-1-z_{j, 3}} m_{j}^{-2-z_{j, 4}} \prod_{i=1}^{t} \frac{\mu\left(\ell_{i}\right) \mu\left(\ell_{i}^{\prime}\right)}{\lambda_{i}} \ell_{i, 1}^{-z_{i, 1}} \ell_{i, 2}^{-z_{i, 2}} \\
= & \sum_{\mathbf{d}, \mathbf{m}, \mathbf{e}, \ell}^{\prime} \prod_{j=t+1}^{t+s} \frac{\mu\left(e_{j}\right) \mu\left(e_{j}^{\prime}\right)}{\epsilon_{j}} e_{j, 1}^{-z_{j, 1}} e_{j, 2}^{-z_{j, 2}} d_{j}^{-1-z_{j, 3}} m_{j}^{-2-z_{j, 4}} \prod_{i=1}^{t} \frac{\mu\left(\ell_{i}\right) \mu\left(\ell_{i}^{\prime}\right)}{\lambda_{i}} \ell_{i, 1}^{-z_{i, 1}} \ell_{i, 2}^{-z_{i, 2}} .
\end{aligned}
$$

Notice that the above is an equation without tildes. We will in the sequel avoid them, observing that for any fixed $\mathbf{u}$, we have

$$
\begin{aligned}
& \sum_{\mathbf{d}, \mathbf{m}, \mathbf{e}, \ell}^{\prime} \prod_{j=t+1}^{t+s} \frac{\mu\left(\tilde{e}_{j}\right) \mu\left(\tilde{e}_{j}^{\prime}\right)}{\epsilon_{j}} \tilde{e}_{j, 1}^{-z_{j, 1}} \tilde{e}_{j, 2}^{-z_{j, 2}} \tilde{d}_{j}^{-1-z_{j, 3}} \tilde{m}_{j}^{-2-z_{j, 4}} \prod_{i=1}^{t} \frac{\mu\left(\ell_{i}\right) \mu\left(\ell_{i}^{\prime}\right)}{\lambda_{i}} \ell_{i, 1}^{-z_{i, 1}} \ell_{i, 2}^{-z_{i, 2}} \\
& =\sum_{\mathbf{d}, \mathbf{m}, \mathbf{e}, \ell}^{\prime} \prod_{j=t+1}^{t+s} \frac{\mu\left(e_{j}\right) \mu\left(e_{j}^{\prime}\right)}{\epsilon_{j}} e_{j, 1}^{-z_{j, 1}} e_{j, 2}^{-z_{j, 2}} d_{j}^{-1-z_{j, 3}} m_{j}^{-2-z_{j, 4}} \prod_{i=1}^{t} \frac{\mu\left(\ell_{i}\right) \mu\left(\ell_{i}^{\prime}\right)}{\lambda_{i}} \ell_{i, 1}^{-z_{i, 1}} \ell_{i, 2}^{-z_{i, 2}} \\
& \times \sum_{\mathbf{v}} \mu\left(v_{j, e}\right) \mu\left(v_{j, e^{\prime}}\right) v_{j, e}^{-z_{j, 1}} v_{j, e^{\prime}}^{-z_{j, 2}} v_{j, d}^{-z_{j, 3}} v_{j, m}^{-z_{j, 4}}
\end{aligned}
$$

where the sum over $\mathbf{v}$ is as usual over vectors $\left(v_{j, x}\right)$ where $v_{j, x} \mid u_{j}$ and $v_{j, x}$ satisfies the same condition on its prime factors as $x$ (all in $\mathcal{P}_{j}$ for $d$ and $e$, all in $\mathcal{Q}_{j}$ for $m$ ).

Next we claim that we can remove the dash on the sum. 
Claim 5. The following equality holds, for any choice of the family $\xi_{j, k}$ in $I=[-\sqrt{\log R}, \sqrt{\log R}]$.

$$
\begin{aligned}
& \sum_{\mathbf{d}, \mathbf{m}, \mathbf{e}, \ell} \prod_{j=t+1}^{t+s} \frac{\mu\left(e_{j}\right) \mu\left(e_{j}^{\prime}\right)}{\epsilon_{j}} e_{j, 1}^{-z_{j, 1}} e_{j, 2}^{-z_{j, 2}} d_{j}^{-1-z_{j, 3}} m_{j}^{-2-z_{j, 4}} \prod_{i=1}^{t} \frac{\mu\left(\ell_{i}\right) \mu\left(\ell_{i}^{\prime}\right)}{\lambda_{i}} \ell_{i}^{-z_{i, 1}} \ell_{i}^{\prime-z_{i, 2}} \\
& =\left(1+O(w(N))^{-1 / 2}\right) \sum_{\mathbf{d}, \mathbf{m}, \mathbf{e}, \ell} \prod_{j=t+1}^{t+s} \frac{\mu\left(e_{j}\right) \mu\left(e_{j}^{\prime}\right)}{\epsilon_{j}} e_{j, 1}^{-z_{j, 1}} e_{j, 2}^{-z_{j, 2}} d_{j}^{-1-z_{j, 3}} m_{j}^{-2-z_{j, 4}} \times \prod_{i \in[t]} \frac{\mu\left(\ell_{i}\right) \mu\left(\ell_{i}^{\prime}\right)}{\lambda_{i}} \ell_{i, 1}^{-z_{i, 1}} \ell_{i, 2}^{-z_{i, 2}} .
\end{aligned}
$$

Proof. The justification is basically the same as for Claim 4, because the claim simply consists in replacing the dashed sum by a complete sum, at the same small cost.

Let us introduce for any $i \in[t]$ and $\mathbf{l}, \boldsymbol{\Xi}$ the notation

$$
V_{i}=V_{i}(\mathbf{l}, \boldsymbol{\Xi})=\frac{\mu\left(\ell_{i}\right) \mu\left(\ell_{i}^{\prime}\right)}{\lambda_{i}} \ell_{i}^{-z_{i, 1}} \ell_{i}^{\prime-z_{i, 2}}
$$

and

$$
V(\mathbf{l}, \Xi)=\prod_{i \in[t]} V_{i}(\mathbf{l}, \Xi)
$$

Similarly, for any $j \in \llbracket t+s ; t+s \rrbracket$ and tuples $\mathbf{u}, \mathbf{v}, \mathbf{d}, \mathbf{m}, \mathbf{e}$ we define

$$
\begin{aligned}
S_{j}(\mathbf{u}, \mathbf{v}, \boldsymbol{\Xi}) & =\frac{2^{s_{j}}}{u_{j}} \mu\left(v_{j, e}\right) \mu\left(v_{j, e^{\prime}}\right) v_{j, e}^{-z_{j, 1}} v_{j, e^{\prime}}^{-z_{j, 2}} v_{j, d}^{-z_{j, 3}} v_{j, m}^{-z_{j, 4}} \\
T_{j}(\mathbf{d}, \mathbf{m}, \mathbf{e}, \boldsymbol{\Xi}) & =\frac{\mu\left(e_{j}\right) \mu\left(e_{j}^{\prime}\right)}{\epsilon_{j}} e_{j}^{-z_{j, 1}} e_{j}^{\prime-z_{j, 2}} d_{j}^{-1-z_{j, 3}} m_{j}^{-2-z_{j, 4}} .
\end{aligned}
$$

Finally we put

$$
S(\mathbf{u}, \mathbf{v}, \boldsymbol{\Xi})=\prod_{j=t+1}^{t+s} S_{j} \quad \text { and } \quad T(\mathbf{d}, \mathbf{m}, \mathbf{e}, \boldsymbol{\Xi})=\prod_{j=t+1}^{t+s} T_{j}
$$

With this notation, one can rewrite (40) as

$$
\left(1+O\left(w^{-1 / 2}\right)\right) \int_{I^{4 s+2 t}} \theta(\boldsymbol{\Xi}) \sum_{\mathbf{u}, \mathbf{v}} S(\mathbf{u}, \mathbf{v}, \boldsymbol{\Xi}) \sum_{\mathbf{d}, \mathbf{m}, \mathbf{e}} T(\mathbf{d}, \mathbf{m}, \mathbf{e}, \boldsymbol{\Xi}) \sum_{\ell} V(\mathbf{l}, \boldsymbol{\Xi}) d \boldsymbol{\Xi} .
$$

Now we show that the error arising from the $O\left(w^{-1 / 2}\right)$ term in (42) is indeed negligible: we must ensure that

$$
w^{-1 / 2} H \sum_{\mathbf{s}, \mathbf{i}} \int_{I^{4 s+2 t}} \theta(\boldsymbol{\Xi}) \sum_{\mathbf{u}, \mathbf{v}} S(\mathbf{u}, \mathbf{v}, \boldsymbol{\Xi}) \sum_{\mathbf{d}, \mathbf{m}, \mathbf{e}} T(\mathbf{d}, \mathbf{m}, \mathbf{e}, \boldsymbol{\Xi}) \sum_{\ell} V(\mathbf{l}, \boldsymbol{\Xi}) d \boldsymbol{\Xi}=o(1) .
$$

This is because on the one hand

$$
\left|\sum_{\mathbf{v}_{\mathbf{j}}} \mu\left(v_{j, e}\right) \mu\left(v_{j, e^{\prime}}\right) v_{j, e}^{-z_{j, 1}} v_{j, e^{\prime}}^{-z_{j, 2}} v_{j, d}^{-z_{j, 3}} v_{j, m}^{-z_{j, 4}}\right| \leq \tau\left(u_{j}\right)^{4}
$$


and

$$
\sum_{\mathbf{s}, \mathbf{i}, \mathbf{u}} \prod_{j=t+1}^{t+s} \frac{2^{s_{j}} \tau\left(u_{j}\right)^{4}}{u_{j}}=O(1)
$$

by similar calculation 8 to the ones of Matthiesen [15, Proof of Proposition 4.2]. And on the other hand, the next claim provides a fitting bound.

Claim 6. We have

$$
\int\left|\theta(\boldsymbol{\Xi}) \sum_{\mathbf{d}, \mathbf{m}, \mathbf{e}} T(\mathbf{d}, \mathbf{m}, \mathbf{e}, \boldsymbol{\Xi}) \sum_{\ell} V(\mathbf{l}, \boldsymbol{\Xi})\right| d \boldsymbol{\Xi}=O\left(1 /(\log R)^{t}\right),
$$

where the integral is over $I^{4 s+2 t}$.

Given that $H=O(\log R)^{t}$, the bound (43) follows from this claim.

Proof. We first replace the sum over $\ell_{i}, \ell_{i}^{\prime}$, for any $i \in[t]$, by a product over primes, using multiplicativity, to get

$$
\sum_{\ell_{i}, \ell_{i}^{\prime}} V_{i}=\sum_{\ell_{i}, \ell_{i}^{\prime}} \frac{\mu\left(\ell_{i}\right) \mu\left(\ell_{i}^{\prime}\right)}{\lambda_{j}} \ell_{i}^{-z_{i, 1}} \ell_{i}^{\prime-z_{i, 2}}=\prod_{s \in \mathcal{P}}\left(1-s^{-1-z_{i, 1}}-s^{-1-z_{i, 2}}+s^{-1-z_{i, 1}-z_{i, 2}}\right) .
$$

Then we notice that for large primes $s$ and complex numbers $z, z^{\prime}$ of positive real part

$$
1-s^{-1-z}-s^{-1-z^{\prime}}+s^{-1-z-z^{\prime}}=\frac{\left(1-s^{-1-z}\right)\left(1-s^{-1-z^{\prime}}\right)}{1-s^{-1-z-z^{\prime}}}+O\left(s^{-2}\right),
$$

so that

$$
\prod_{s \in \mathcal{P}}\left(1-s^{-1-z_{j, 1}}-s^{-1-z_{j, 2}}+s^{-1-z_{j, 1}-z_{j, 2}}\right) \ll \prod_{s \in \mathcal{P}} \frac{\left(1-s^{-1-z}\right)\left(1-s^{-1-z^{\prime}}\right)}{1-s^{-1-z-z^{\prime}}} .
$$

Finally we recall that the $\zeta$ function is defined for $\Re z>1$ by

$$
\zeta(z)=\sum_{n \geq 1} n^{-z}=\prod_{p}\left(1-p^{-z}\right)^{-1}
$$

and satisfies

$$
\zeta(z)=\frac{1}{z-1}+O(1)
$$

for values of $z$ near 1 . From this fact, a quick computation yields

$$
\prod_{s \in \mathcal{P}} \frac{\left(1-s^{-1-z}\right)\left(1-s^{-1-z^{\prime}}\right)}{1-s^{-1-z-z^{\prime}}} \ll \frac{z z^{\prime}}{z+z^{\prime}}
$$

whence the bound

$$
\prod_{s \in \mathcal{P}}\left(1-s^{-1-z_{i, 1}}-s^{-1-z_{i, 2}}+s^{-1-z_{i, 1}-z_{i, 2}}\right) \ll \frac{z_{i, 1} z_{i, 2}}{z_{i, 1}+z_{i, 2}} .
$$

\footnotetext{
${ }^{8}$ The main ingredients are the easy observation that any $u \in U(i, s)$ has $2^{m_{0}(i, s)}$ divisors and the bound $\sum_{u \in U(i, s)} u^{-1} \leq\left(\sum_{p \in I_{i}} p^{-1}\right)^{m_{0}} \ll(\log 2)^{m_{0}}$, where $I_{i}=\left[N^{2^{-i-1}}, N^{2^{-i}}\right]$.
} 
for any $i \in[t]$ and $\xi_{i, k} \in I$ (for $k=1,2$ ) and the corresponding $z_{i, k}$. Similarly, for any $j \in\{t+1, \ldots, t+s\}$

$$
\begin{aligned}
\sum_{d_{j}, m_{j}, e_{j}, e_{j}^{\prime}} \frac{\mu\left(e_{j}\right) \mu\left(e_{j}^{\prime}\right)}{\epsilon_{j}} e_{j}^{-z_{j, 1}} e_{j}^{\prime-z_{j, 2}} d_{j}^{-1-z_{j, 3}} m_{j}^{-2-z_{j, 4}} & =\prod_{q \in \mathcal{Q}_{j}}\left(1-q^{-1-z_{j, 1}}-q^{-1-z_{j, 2}}+q^{-1-z_{j, 1}-z_{j, 2}}\right) \\
& \prod_{r \in \mathcal{Q}_{j}}\left(1-r^{-2-z_{j, 4}}\right)^{-1} \prod_{p \in \mathcal{P}_{j}}\left(1-p^{-1-z_{j, 3}}\right)^{-1} .
\end{aligned}
$$

Notice that the product in $r$ is a convergent product, bounded by a constant when $z_{j, 4}$ varies in the permitted range.

Given that $\mathcal{P}_{j}$ and $\mathcal{Q}_{j}$ each have density $1 / 2$ among the primes, we can write

$$
\sum_{q \in \mathcal{P}_{j}} q^{-1-z}=\frac{1}{2} \log \frac{1}{z}+O(1)
$$

for $\Re z>09$ This provides a bound for the product (46), similar to the one in (45), namely

$$
\begin{aligned}
& \prod_{q \in \mathcal{Q}_{j}}\left(1-q^{-1-z_{j, 1}}-q^{-1-z_{j, 2}}+q^{-1-z_{j, 1}-z_{j, 2}}\right) \prod_{r \in \mathcal{Q}_{j}}\left(1-r^{-2-z_{j, 4}}\right)^{-1} \prod_{p \in \mathcal{P}_{j}}\left(1-p^{-1-z_{j, 3}}\right)^{-1} \\
& \ll\left|z_{j, 1}\right|^{1 / 2}\left|z_{j, 2}\right|^{1 / 2}\left|z_{j, 1}+z_{j, 2}\right|^{-1 / 2}\left|z_{j, 3}\right|^{-1 / 2}
\end{aligned}
$$

Recall that $z_{j, k}=\left(1+\xi_{j, k}\right)(\log R)^{-1}$, thus $\left|z_{j, k}\right| \leq\left(1+\left|\xi_{j, k}\right|\right)(\log R)^{-1}$ by triangle inequality, and $\left|z_{j, 1}+z_{j, 2}\right|^{-1} \leq \log R$ for any $j \in[t+s]$. Moreover, (37) yields

$$
\theta(\boldsymbol{\Xi})=O_{A}\left(\prod_{j, k}\left(1+\left|\xi_{j, k}\right|\right)^{-A}\right) .
$$

Multiplying all these bounds, we find that the integrand in (44) is bounded by

$$
\begin{aligned}
& \prod_{i=1}^{t}\left|z_{i, 2}\right|\left|z_{i, 1}\right|\left|z_{i, 1}+z_{i, 2}\right|^{-1} \prod_{j=t+1}^{t+s}\left|z_{j, 1}\right|^{1 / 2}\left|z_{j, 2}\right|^{1 / 2}\left|z_{j, 1}+z_{j, 2}\right|^{-1 / 2}\left|z_{j, 3}\right|^{-1 / 2} \prod_{j, k}\left(1+\left|\xi_{j, k}\right|\right)^{-A} \\
& \ll(\log R)^{-t}\left(\prod_{i=1}^{t}\left(1+\left|\xi_{i, 1}\right|\right)\left(1+\left|\xi_{i, 2}\right|\right)\right)^{1-A}\left(\prod_{j=t+1}^{t+s}\left(1+\left|\xi_{j, 1}\right|\right)\left(1+\left|\xi_{j, 2}\right|\right)\right)^{1 / 2-A} \\
& \ll(\log R)^{-t} \prod_{j, k}\left(1+\left|\xi_{j, k}\right|\right)^{-A / 2}
\end{aligned}
$$

when $A$ is large enough (for the last step). This last product is certainly integrable as soon as $A>2$, so the final expression is $O\left((\log R)^{-t}\right)$ as claimed.

\footnotetext{
${ }^{9}$ This amounts to saying that if a set of primes has a natural density, it has a Dirichlet density which is equal to its natural density.
} 
We now study the main term of (42). We can again swap summation and integration using Fubini's theorem. Using separation of variables, we transform the main term of (42) into

$$
\sum_{\mathbf{u}, \mathbf{v}, \mathbf{d}, \mathbf{e}, \mathbf{m}, \ell} \prod_{i=1}^{t} \int_{I^{2}} V_{i} \theta\left(\xi_{i, 1}\right) \theta\left(\xi_{i, 2}\right) d \xi_{i, 1} d \xi_{i, 2} \prod_{j=t+1}^{t+s} \int_{I^{4}} S_{j} T_{j} \prod_{k \in[4]} \theta\left(\xi_{j, k}\right) d \xi_{j, k}
$$

It is now time to undo the truncation to $I$ in these integrals, in order to be able to collapse them into factors of $\chi$. The error term arising from the removal of this truncation is the same as the one introduced by the truncation, so it can be subsumed into the $o(1)$ of (23) $)$. Thus, up to an error term $E_{\mathbf{i}, \mathbf{s}}$ satisfying $(\log R)^{t} \sum_{\mathbf{i}, \mathbf{s}} E_{\mathbf{i}, \mathbf{s}}=o(1)$, the expression (47) is equal to

$$
\begin{aligned}
& \sum_{\mathbf{u}, \mathbf{v}, \mathbf{d}, \mathbf{e}, \mathbf{m}, \ell} \prod_{i=1}^{t} \frac{\mu\left(\ell_{i, 1}\right) \mu\left(\ell_{i, 2}\right)}{\lambda_{i}} \prod_{k=1,2} \chi\left(\frac{\log \ell_{i, k}}{\log R}\right) \\
& \quad \prod_{j=t+1}^{t+s} \frac{2^{s_{j}} \tau\left(u_{j}\right)}{u_{j}} \frac{\mu\left(e_{j} v_{j, e}\right) \mu\left(e_{j}^{\prime} v_{j, e^{\prime}}\right)}{d_{j} m_{j}^{2} \epsilon_{j}} \chi\left(\frac{\log d_{j} v_{j, d}}{\log R}\right) \chi\left(\frac{\log m_{j} v_{j, m}}{\log R}\right) \prod_{k=1,2} \chi\left(\frac{\log e_{j, k} v_{j, e_{k}}}{\log R}\right) .
\end{aligned}
$$

Interchanging summation and multiplication, we find that, up to error terms of the desired magnitude $\left(O_{D}\left(\frac{N^{d-1+O_{D}(\gamma)}}{\operatorname{Vol}(K)}\right)\right.$ in Claims 1 and 2, various $o(1)$ throughout the proof $), \Omega$ equals

$$
\begin{aligned}
& \prod_{j=t+1}^{t+s} C_{D_{j}, \gamma}^{-1} \sum_{s_{j}, i_{j}, u_{j}, v_{j}} \sum_{d_{j}, m_{j}, e_{j}, e_{j}^{\prime}} \frac{2^{s_{j}}}{u_{j}} \frac{\mu\left(e_{j} v_{j, e}\right) \mu\left(e_{j}^{\prime} v_{j, e^{\prime}}\right)}{d_{j} m_{j}^{2} \epsilon_{j}} \prod_{x \in\left\{d, m, e, e^{\prime}\right\}} \chi\left(\frac{\log x_{j} v_{j, x}}{\log R}\right) \\
& \quad \times \prod_{i \in[t]}\left(\log R \frac{\phi(W)}{W} \sum_{\ell_{i}, \ell_{i}^{\prime}} \frac{\mu\left(\ell_{i}\right) \mu\left(\ell_{i}^{\prime}\right)}{\lambda} \prod_{x \in\left\{\ell_{i}, \ell_{i}^{\prime}\right\}} \chi\left(\frac{\log x}{\log R}\right)\right),
\end{aligned}
$$

which is a product of $t+s$ factors, independent of the system of linear forms. Hence the $j$ th factor, for $j \in[t+s]$, is also the main term of the average of the $j$ th pseudorandom majorant for the trivial system $\Phi: \mathbb{Z} \rightarrow \mathbb{Z}, n \mapsto n$. Now because of the properties of the Green-Tao and the Matthiesen majorant, described in subsections 3.2.1 and 3.2.2 respectively, these averages are $1+o(1)$, whence the result.

\section{Appendix C. Volume paCking arguments And local Divisor Density}

In this appendix, we shall collect some frequently used facts concerning the number of solutions to a system of linear equations in a convex set of $\mathbb{R}^{d}$ and in $(\mathbb{Z} / m \mathbb{Z})^{d}$. We first recall a lemma already stated earlier but particularly relevant here, borrowed from Green and Tao [8, Appendix A]. 
Lemma C.1. Let $K \subset[0, N]^{d}$ be a convex body of $\mathbb{R}^{d}$. Then

$$
\left|K \cap \mathbb{Z}^{d}\right|=\sum_{n \in K \cap Z^{d}}=\operatorname{Vol}(K)+O_{d}\left(N^{d-1}\right) .
$$

We recall the definition of the local divisor density and we mention some useful properties.

Definition C.1. For a given system of affine-linear forms $\Psi=\left(\psi_{1}, \ldots, \psi_{t}\right): \mathbb{Z}^{d} \rightarrow \mathbb{Z}^{t}$, positive integers $d_{1}, \ldots, d_{t}$ of lcm $m$, define the local divisor density by

$$
\alpha_{\Psi}\left(d_{1}, \ldots, d_{t}\right)=\mathbb{E}_{n \in(\mathbb{Z} / m \mathbb{Z})^{d}} \prod_{i=1}^{t} 1_{\psi_{i}(n) \equiv 0 \bmod d_{i}} .
$$

The following lemma is borrowed from Matthiesen [16, Lemma 9.3].

Lemma C.2. Let $K \subset[-B, B]^{d}$ be a convex body and $\Psi$ a system of affine-linear forms, and let $d_{1}, \ldots, d_{t}$ be integers of lcm $m$. Then

$$
\sum_{n \in \mathbb{Z}^{d} \cap K} \prod_{d_{i} \mid \psi_{i}(n)}=\operatorname{Vol}(K) \alpha_{\Psi}\left(d_{1}, \ldots, d_{t}\right)+O\left(B^{d-1} m\right) .
$$

We shall try to bound $\alpha_{\Psi}\left(p^{a_{1}}, \ldots, p^{a_{t}}\right)$. To this aim, we state a version of Hensel's lemma in several variables.

Lemma C.3. Let $Q \in \mathbb{Z}\left[X_{1}, \ldots, X_{d}\right]$, $p$ be a prime and $k \geq 1$ an integer and $x \in\left(\mathbb{Z} / p^{k} \mathbb{Z}\right)^{d}$ such that $Q(x) \equiv 0 \bmod p^{k}$ and

$$
\overrightarrow{\operatorname{grad}} Q(x)=\left(\frac{\partial Q}{\partial x_{1}}, \ldots, \frac{\partial Q}{\partial x_{d}}\right)(x) \neq 0 \bmod p .
$$

Then there exist precisely $p^{d-1}$ vectors $y \in\left(\mathbb{Z} / p^{k+1} \mathbb{Z}\right)^{d}$ such that $x \equiv y \bmod p^{k}$ and $Q(y) \equiv$ $0 \bmod p^{k+1}$.

Proof. Let $y \in\left(\mathbb{Z} / p^{k+1} \mathbb{Z}\right)^{d}$ satisfy $x \equiv y \bmod p^{k}$; in other words, $y=x+p^{k} z$ for some uniquely determined $z \in(\mathbb{Z} / p \mathbb{Z})^{d}$. Here we treat $x \in\left(\mathbb{Z} / p^{k} \mathbb{Z}\right)^{d}$ as an element of $\left(\mathbb{Z} / p^{k+1} \mathbb{Z}\right)^{d}$ by using the canonical injection. We then treat $Q(x)$ as an element of $\mathbb{Z} / p^{k+1} \mathbb{Z}$ congruent to $0 \bmod p^{k}$ and put $Q(x)=p^{k} a$ with $a \in \mathbb{Z} / p \mathbb{Z}$. Then Taylor's formula ensures that

$$
Q(y) \equiv Q(x)+p^{k} \overrightarrow{\operatorname{grad}} Q(x) \cdot z \equiv p^{k}(a+\overrightarrow{\operatorname{grad}} Q(x) \cdot z) \bmod p^{k+1} .
$$

So $Q(y) \equiv 0 \bmod p^{k+1}$ is equivalent to $a+\overrightarrow{\operatorname{grad}} Q(x) \cdot z \equiv 0 \bmod p$. As $\overrightarrow{\operatorname{grad}} Q(x)$ is not zero modulo $p$, this imposes a nontrivial affine equation on $z$ in the vector space $\mathbb{F}_{p}^{d}$, so $z$ is constrained to lie in a $(d-1)$-dimensional affine $\mathbb{F}_{p}$-subspace, which has $p^{d-1}$ elements, hence the conclusion.

As an application, we prove the following statement.

Corollary C.4. Let $\psi$ be an affine-linear form in $d$ variables, and let $p$ be a prime such that $\psi$ is not the trivial form modulo $p$. Then for any $m \geq 1$

$$
\alpha_{m}=\alpha_{\psi}\left(p^{m}\right)=\mathbb{E}_{n \in\left(\mathbb{Z} / p^{m} \mathbb{Z}\right)^{d}} 1_{p^{m} \mid \psi(n)}=\mathbb{P}_{n \in\left(\mathbb{Z} / p^{m} \mathbb{Z}\right)^{d}}(\psi(n)=0) \leq p^{-m} .
$$


Remark C.1. If $\psi=p^{k} \psi^{\prime}$ and $\psi^{\prime}$ is not the trivial form modulo $p$, this corollary provides for $m \geq k$ the bound $\alpha_{\psi}\left(p^{m}\right) \leq p^{k-m} \ll p^{-m}$.

Proof. If $n \in\left(\mathbb{Z} / p^{m} \mathbb{Z}\right)^{d}$ satisfies $\psi(n) \equiv 0 \bmod p^{m}$, then in particular $\tilde{\psi}(\tilde{n}) \equiv 0 \bmod p$, where $\sim$ is the reduction modulo $p$, which imposes that $\tilde{n}$ lies in ker $\tilde{\psi}$. By assumption, $\tilde{\psi} \neq 0$. If its linear part is 0 , then its constant part is nonzero, thus $\operatorname{ker} \tilde{\psi}=\emptyset$ and $\alpha_{m}=0$. Otherwise, the linear part is nonzero modulo $p$, and then $\operatorname{ker} \tilde{\psi}$ is an affine $\mathbb{F}_{p}$-hyperplane, thus has $p^{d-1}$ elements. Let us prove the proposition by induction on $m$. For $m=1$, we have just proved the result. Suppose now that $\alpha_{m} \leq p^{-m}$ for some $m \geq 1$. Because of the assumption above, $\overrightarrow{\operatorname{grad}} \psi$ is a constant vector which is nonzero modulo $p$. Applying Lemma C.3 for $k=m$, we find that each zero modulo $p^{m}$ of $\psi$ gives rise to exactly $p^{d-1}$ zeros modulo $p^{m+1}$, which proves that $\alpha_{m+1} \leq p^{-(m+1)}$. This concludes the induction step and the proof.

Exploiting this corollary, we can now prove a bound on more general local densities.

Proposition C.5. Let $\Psi=\left(\psi_{1}, \ldots, \psi_{t}\right)$ be a system of integral affine linear forms in $d$ variables and $p$ be a prime so that the system reduced modulo $p$ is of finite complexity, i.e. no two of the forms are affinely related modulo $p$. Then

$$
\alpha=\alpha_{\Psi}\left(p^{a_{1}}, \ldots, p^{a_{t}}\right) \leq p^{-\max _{i \neq j}\left(a_{i}+a_{j}\right)} .
$$

Proof. If all $a_{i}$ are zero, the result is trivial, so let $m=\max a_{i}$ and suppose $m \geq 1$; let $i<j$ be such that $a_{i}+a_{j}$ is maximal (in particular, it is at least $m$ ). Suppose first that either $a_{i}$ or $a_{j}$ is 0 . Without loss of generality, suppose $a_{i}=0$ and $a_{j} \neq 0$. Then for $n \in\left(\mathbb{Z} / p^{m} \mathbb{Z}\right)^{d}$ to satisfy $\psi_{k}(n) \equiv 0 \bmod p^{a_{k}}$ for all $k=1, \ldots, t$, we must have in particular $\tilde{\psi}_{j}(\tilde{n}) \equiv 0 \bmod p^{a_{j}}$, and using Corollary C.4, we find that

$$
\alpha=\mathbb{E}_{n \in\left(\mathbb{Z} / p^{m} \mathbb{Z}\right)^{d}} \prod_{i \in[t]} 1_{p^{a_{i} \mid \phi_{i}(n)}} \leq \mathbb{E}_{n \in\left(\mathbb{Z} / p^{a_{j}} \mathbb{Z}\right)^{d}} 1_{p^{a_{j}} \mid \phi_{j}(n)}=p^{-a_{j}}=p^{-\max _{i \neq j}\left(a_{i}+a_{j}\right)} .
$$

Now suppose $1 \leq a_{i} \leq a_{j}$. Then for $n \in\left(\mathbb{Z} / p^{m} \mathbb{Z}\right)^{d}$ to satisfy $\psi_{k}(n) \equiv 0 \bmod p^{a_{k}}$ for all $k=1, \ldots, t$, we must have in particular $\tilde{\psi}_{i}(\tilde{n}) \equiv \tilde{\psi}_{j}(\tilde{n}) \equiv 0 \bmod p$. We can again suppose that $\tilde{\psi}_{j}$ as well as $\tilde{\psi}_{i}$ have linear parts which are not 0 modulo $p$, otherwise $\alpha=0$. This imposes that $\tilde{n}$ lies in the intersection of two affine $\mathbb{F}_{p}$-hyperplanes, namely $\operatorname{ker} \tilde{\psi}_{i}$ and ker $\tilde{\psi}_{j}$ which are distinct because these forms are affinely independent modulo $p$. This intersection is empty (and then $\alpha=0$ ) if and only if these hyperplanes are parallel (hence the linear parts of $\psi_{j}$ and $\psi_{i}$ are proportional modulo $p$ ). So let us suppose that the linear parts are not proportional modulo $p$, which amounts to saying that the constant vectors $\overrightarrow{\operatorname{grad}} \tilde{\psi}_{j}, \overrightarrow{\operatorname{grad}} \tilde{\psi}_{i} \in(\mathbb{Z} / p \mathbb{Z})^{d}$ are not proportional. Now we use induction on $m \geq 1$ to show that

$$
\beta_{m}=\mathbb{P}_{n \in\left(\mathbb{Z} / p^{m} \mathbb{Z}\right)^{d}}\left(\psi_{i}(n) \equiv \psi_{j}(n) \equiv 0 \bmod p^{m}\right) \leq p^{-2 m}
$$

For $m=1$, what we have seen above implies that $\beta_{1}=0$ or $\beta_{1}=p^{-2}$ (the intersection of two nonparallel affine hyperplanes of $\mathbb{F}_{p}^{d}$ is an affine subspace of dimension $d-2$, so its cardinality is $p^{d-2}$ ), so the statement is true. Suppose now that for some $m \geq 1$ we have $\beta_{m} \leq p^{-2 m}$. 
If $x \in\left(\mathbb{Z} / p^{m} \mathbb{Z}\right)^{d}$ satisfies $\psi_{i}(x) \equiv \psi_{j}(x) \equiv 0 \bmod p^{m}$ and if $y=x+p^{m} z \in\left(\mathbb{Z} / p^{m+1} \mathbb{Z}\right)^{d}$ for some $z \in(\mathbb{Z} / p \mathbb{Z})^{d}$ satisfies $\psi_{i}(y) \equiv \psi_{j}(y) \equiv 0 \bmod p^{m+1}$, then following the proof of Lemma C.3, we infer that $z$ has to satisfy two affine equations

$$
a+\overrightarrow{\operatorname{grad}} \psi_{i} \cdot z \equiv 0 \bmod p \quad \text { and } \quad a+\overrightarrow{\operatorname{grad}} \psi_{j} \cdot z \equiv 0 \bmod p .
$$

This forces $z$ to lie in the intersection of two nonparallel affine $\mathbb{F}_{p}$-hyperplanes of $\mathbb{F}_{p}^{d}$ (they are nonparallel because we supposed that the gradients were not proportional). Hence for a fixed $x$ as above, there are $p^{d-2}$ such $y$, so finally $\beta_{m+1}=p^{d-2} \beta_{m}$ whence the conclusion. In particular, putting $m=a_{i}$, we have that $\mathbb{E}_{n \in\left(\mathbb{Z} / p^{\left.a_{i} \mathbb{Z}\right)^{d}}\right.} 1_{\phi_{i}(n) \equiv \phi_{j}(n) \equiv 0 \bmod p^{a_{i}}} \leq p^{-2 a_{i}}$. It remains to induct on $a_{j}-a_{i} \geq 0$ using Lemma C.3 in order to find that

$$
\mathbb{E}_{n \in\left(\mathbb{Z} / p^{a_{j}} \mathbb{Z}\right)^{d}} 1_{p^{a_{i} \mid \phi_{i}(n)}} 1_{p^{a_{j}} \mid \phi_{j}(n)} \leq p^{-\left(a_{i}+a_{j}\right)},
$$

which implies the desired result.

We prove another statement which is helpful during the proof of the linear forms conditions (Appendix B).

Proposition C.6. Let $\Phi: \mathbb{Z}^{d} \rightarrow \mathbb{Z}^{t}$ be a system of affine-linear forms. Let $p$ be a prime such that the reduction modulo $p$ of the system is of finite complexity. Let $K \subset[-B, B]^{d}$ be a convex body. Then

$$
\sum_{n \in K \cap \mathbb{Z}^{d}} 1_{p^{2} \mid \prod_{i \in[t]} \phi_{i}(n)} \ll_{t} p^{-2} \operatorname{Vol}(K)+B^{d-1} p^{2}
$$

Proof. First, we remark that $p^{2} \mid \prod_{i \in[t]} \phi_{i}(n)$ implies that either there exists $i \in[t]$ such that $p^{2} \mid \phi_{i}(n)$ or there exist $i \neq j$ such that $p \mid \phi_{i}(n)$ and $p \mid \phi_{j}(n)$. Hence

$$
\sum_{n \in K \cap \mathbb{Z}^{d}} 1_{p^{2} \mid \prod_{i \in[t]} \phi_{i}(n)} \leq \sum_{i \in[t]} \sum_{n \in K \cap \mathbb{Z}^{d}} 1_{p^{2} \mid \phi_{i}(n)}+\sum_{i \neq j} \sum_{n \in K \cap \mathbb{Z}^{d}} 1_{\phi_{i}(n) \equiv \phi_{j}(n) \equiv 0 \bmod p} .
$$

Now for any $i \in[t]$ we apply Lemma C.2 which implies

$$
\sum_{n \in K \cap \mathbb{Z}^{d}} 1_{p^{2} \mid \phi_{i}(n)}=\operatorname{Vol}(K) \alpha_{\phi_{i}}\left(p^{2}\right)+O\left(B^{d-1} p^{2}\right)
$$

and for any $i \neq j$

$$
\sum_{n \in K \cap \mathbb{Z}^{d}} 1_{\phi_{i}(n) \equiv \phi_{j}(n) \equiv 0 \bmod p}=\operatorname{Vol}(K) \alpha_{\phi_{i}, \phi_{j}}(p, p)+O\left(B^{d-1} p\right) .
$$

But the assumption of finite complexity modulo $p$ means that we may invoke Proposition C.5, which implies that $\alpha_{\phi_{i}}\left(p^{2}\right) \leq p^{-2}$ and that $\alpha_{\phi_{i}, \phi_{j}}(p, p) \leq p^{-2}$. The result then follows. 


\section{REFERENCES}

[1] A. Balog. The Hardy-Littlewood k-tuple conjecture on average. Analytic Number Theory, Birkhäuser, 1990, 47-75.

[2] T. Browning and L. Matthiesen. Norm forms for arbitrary number fields as products of linear polynomials, preprint. arXiv:1307.7641, July 2013.

[3] D. Conlon, J. Fox, and Y. Zhao. The Green-Tao theorem: an exposition. EMS Surv. Math. Sci., $1(2): 249-282,2014$.

[4] P. Erdös. On the sum $\sum_{k=1}^{x} d(f(k))$. J. London Math. Soc., 27:7-15, 1952.

[5] E. Fouvry. Sur le problème des diviseurs de Titchmarsh. J. Reine Angew. Math., 357:51-76, 1984.

[6] B. Green. On arithmetic structures in dense sets of integers. Duke Math. J., 114(2):215-238, 2002.

[7] B. Green and T. Tao. The primes contain arbitrarily long arithmetic progressions. Ann. of Math, pages $481-547$.

[8] B. Green and T. Tao. Linear equations in primes. Ann. of Math. (2), 171(3):1753-1850, 2010.

[9] B. Green and T. Tao. The Möbius function is strongly orthogonal to nilsequences. Ann. of Math. (2), 175(2):541-566, 2012.

[10] B. Green, T. Tao, and T. Ziegler. An inverse theorem for the Gowers $U^{s+1}[N]$-norm. Ann. of Math. (2), 176(2):1231-1372, 2012.

[11] H. Iwaniec and E. Kowalski. Analytic number theory, volume 53 of American Mathematical Society Colloquium Publications. American Mathematical Society, Providence, RI, 2004.

[12] T.H. Lê and J. Wolf. Polynomial configurations in the primes. Int. Math. Res. Not. IMRN, (23):6448$6473,2014$.

[13] Ju. V. Linnik. The dispersion method in binary additive problems. Translated by S. Schuur. American Mathematical Society, Providence, R.I., 1963.

[14] L. Matthiesen. Linear correlations of multiplicative functions. arXiv:1606.04482, June 2016.

[15] L. Matthiesen. Correlations of the divisor function. Proc. Lond. Math. Soc, 104:827-858., 2012.

[16] L. Matthiesen. Linear correlations amongst numbers represented by positive definite binary quadratic forms. Acta Arith, 154:235-306, 2012.

[17] T. Tao and T. Ziegler. The primes contain arbitrarily long polynomial progressions. Acta Math., 201(2):213-305, 2008.

[18] T. Tao and T. Ziegler. Narrow progressions in the primes, preprint. arXiv:1409.1327, September 2014.

[19] T. Tao and T. Ziegler. Polynomial patterns in the primes, preprint. arXiv:1603.07817, March 2016.

[20] E.C. Titchmarsh. A divisor problem. Rend. di Palermo, 54:414-429, 1931.

School of Mathematics, University of Bristol, Bristol BS8 1TW, United Kingdom

E-mail address: pb14917@bristol.ac.uk 